TRANSACTIONS OF THE

AMERICAN MATHEMATICAL SOCIETY

Volume 365, Number 5, May 2013, Pages 2317-2339

S 0002-9947(2012)05671-6

Article electronically published on December 12, 2012

\title{
DECAY ESTIMATES FOR RIVIÈRE'S EQUATION, WITH APPLICATIONS TO REGULARITY AND COMPACTNESS
}

\author{
BEN SHARP AND PETER TOPPING
}

\begin{abstract}
We derive a selection of energy estimates for a generalisation of a critical equation on the unit disc in $\mathbb{R}^{2}$ introduced by Rivière. Applications include sharp regularity results and compactness theorems which generalise a large amount of previous geometric PDE theory, including some of the theory of harmonic and almost-harmonic maps from surfaces.
\end{abstract}

\section{INTRODUCTION}

Suppose $u \in W^{1,2}\left(B_{1}, \mathbb{R}^{m}\right)$ is a weak solution to

$$
-\Delta u=\Omega . \nabla u,
$$

where here and throughout this paper $B_{1}$ is the unit disc in $\mathbb{R}^{2}, \Omega \in L^{2}\left(B_{1}, s o(m) \otimes\right.$ $\mathbb{R}^{2}$ ), and we are using the notation $[\Omega . \nabla u]^{i}=\left\langle\Omega_{j}^{i}, \nabla u^{j}\right\rangle$. This equation, first considered in this generality by Rivière [12, generalises a number of interesting equations appearing naturally in geometry, including the harmonic map equation, the $H$-surface equation and, more generally, the Euler-Lagrange equation of any conformally invariant elliptic Lagrangian which is quadratic in the gradient. A central issue is the regularity of $u$ implied by virtue of its satisfying equation (11). A priori, the right-hand side of the equation looks like quite a general $L^{1}$ function, and standard elliptic regularity theory does not seem to help. However, Rivière 12. showed that any solution must necessarily be continuous and even in $W^{2,1+\epsilon}$ for some $\epsilon>0$ [13], thus generalising the famous regularity theory of Hélein [8], for example. In most known interesting special cases of this equation, one happens to know that $|\Omega|$ can be estimated linearly in terms of $|\nabla u|$; i.e. we have $|\Omega . \nabla u| \leq$ $C|\nabla u|^{2}$ and then a standard bootstrapping argument can be applied to improve the regularity of $u$ to $W^{2, p}$ for any $p<\infty$. Moreover typically in such cases $\Omega$ can be viewed as a smooth function of $u$ and $\nabla u$, at which point we can conclude that $u$ is smooth using Schauder theory (and the embedding $W^{2, p}\left(\mathbb{R}^{2}\right) \hookrightarrow C^{1,1-\frac{2}{p}}\left(\mathbb{R}^{2}\right)$ for $p>2$ ).

In this paper we investigate what sort of regularity and compactness properties we can deduce for solutions of the general equation (11), and even more general inhomogeneous equations with the same special structure. It is easy to convince oneself that it is unreasonable to expect regularity better than $W^{2,2}$ in general. However, we will show that we do have regularity up to this level, or the best possible regularity when there is an inhomogeneity.

Received by the editors April 1, 2011.

2010 Mathematics Subject Classification. Primary 42B37, 35A23, 35B65.

(C)2012 the authors 
Theorem 1.1. Suppose that $u \in W^{1,2}\left(B_{1}, \mathbb{R}^{m}\right)$ is a weak solution on the unit disc in $\mathbb{R}^{2}$ to

$$
-\Delta u=\Omega . \nabla u+f, \quad f \in L^{p}\left(B_{1}, \mathbb{R}^{m}\right),
$$

where $\Omega \in L^{2}\left(B_{1}, s o(m) \otimes \mathbb{R}^{2}\right)$ and $p \in(1,2)$. Then $u \in W_{\text {loc }}^{2, p}\left(B_{1}\right)$. In particular, if $f \equiv 0$, then $u \in W_{\text {loc }}^{2, p}$ for all $p \in[1,2)$ and $u \in W_{\text {loc }}^{1, q}$ for all $q \in[1, \infty)$.

Moreover, for $U \Subset B_{1}$, there exist $\eta_{0}=\eta_{0}(p, m)>0$ and $C=C(p, m, U)<\infty$ so that if $\|\Omega\|_{L^{2}\left(B_{1}\right)} \leq \eta_{0}$, then

$$
\|u\|_{W^{2, p}(U)} \leq C\left(\|f\|_{L^{p}\left(B_{1}\right)}+\|u\|_{L^{1}\left(B_{1}\right)}\right) .
$$

This theorem omits the borderline case $p=2$ for good reason; even in the case that $f \equiv 0$, one can find solutions so that $u$ is neither $W^{2,2}$ nor Lipschitz. Moreover, examples with $f \equiv 0$ show that the first derivatives of $u$ need not even lie in $B M O$, and (consequently) the second derivatives need not even lie in the Lorentz space $L^{2, \infty}$ (see Appendices $\mathrm{A} .2$ and $\mathrm{A.3}$ for definitions if necessary).

As a corollary of our theorem, we see that $f \in L^{p}$ implies that $u$ lies in $C^{0,2\left(1-\frac{1}{p}\right)}$, hence recovering a result of Rupflin [14] in the case of two-dimensional domains. Rivière has informed us that our regularity assertion in the particular case $f \equiv 0$ will also be made in the final version of [13], based on a different proof.

We remark that the estimate (3) fails without the smallness of $\Omega$ hypothesis. More precisely, there exist a sequence $\Omega_{k} \in L^{2}\left(B_{1}, s o(m) \otimes \mathbb{R}^{2}\right)$ uniformly bounded in $L^{2}$, and a sequence of weak solutions $u_{k} \in W^{1,2}\left(B_{1}, \mathbb{R}^{m}\right)$ to the equation

$$
-\Delta u_{k}=\Omega_{k} \cdot \nabla u_{k},
$$

uniformly bounded in $W^{1,2}$, such that $u_{k}$ is unbounded in any $W^{2, p}$ space with $p \in(1,2)$. (A sequence of harmonic maps undergoing bubbling would provide an example, for instance the sequence given in Section 8,

Estimate (3) implies that for any sequence $\Omega_{k} \in L^{2}\left(B_{1}, s o(m) \otimes \mathbb{R}^{2}\right)$ with $\left\|\Omega_{k}\right\|_{L^{2}\left(B_{1}\right)} \leq \eta_{0}$, and any sequence of weak solutions $u_{k} \in W^{1,2}\left(B_{1}, \mathbb{R}^{m}\right)$ to the equation

$$
-\Delta u_{k}=\Omega_{k} . \nabla u_{k}+f_{k}
$$

with $u_{k}$ uniformly bounded in $W^{1,2}$ and $f_{k}$ uniformly bounded in some space $L^{p}$ for $p \in(1,2)$, we may deduce that $u_{k}$ is locally uniformly bounded in $W^{2, p}$. By the theorem of Rellich-Kondrachov, we can deduce that $u_{k}$ is precompact in $W^{1, t}\left(B_{1 / 2}\right)$ for any $t<\frac{2 p}{2-p}$.

In this paper, we work somewhat harder to prove a stronger compactness result, extending a recent theorem of $\mathrm{Li}$ and $\mathrm{Zhu}$ [11, in which we assume merely that the inhomogeneous terms $f_{k}$ are bounded in $L \ln L$ (a space larger than any of the $L^{p}$ spaces with $p>1$, but slightly smaller than $L^{1}$; see Appendix A.3 for more information on this space, and definitions, if necessary).

Theorem 1.2 (Compactness). Suppose that we have a sequence $\left\{u_{n}\right\} \subset W^{1,2}\left(B_{1}\right.$, $\mathbb{R}^{m}$ ) of weak solutions to

$$
-\Delta u_{n}=\Omega_{n} \cdot \nabla u_{n}+f_{n}
$$

on the unit disc in $\mathbb{R}^{2}$, where $\left\{\Omega_{n}\right\} \subset L^{2}\left(B_{1}\right.$, so $\left.(m) \otimes \mathbb{R}^{2}\right)$ and $\left\{f_{n}\right\} \subset L \ln L\left(B_{1}, \mathbb{R}^{m}\right)$. Suppose also that there exists $\Lambda<\infty$ such that

$$
\left\|u_{n}\right\|_{L^{1}\left(B_{1}\right)}+\left\|f_{n}\right\|_{L \ln L\left(B_{1}\right)} \leq \Lambda .
$$


Then there exist an $\eta_{2}=\eta_{2}(m)>0$ and $u \in W_{l o c}^{1,2}\left(B_{1}, \mathbb{R}^{m}\right)$ such that if $\left\|\Omega_{n}\right\|_{L^{2}\left(B_{1}\right)}$ $\leq \eta_{2}$, then after passing to a subsequence,

$$
\lim _{n \rightarrow \infty}\left\|u_{n}-u\right\|_{W^{1,2}\left(B_{1 / 2}\right)}=0 .
$$

We will show in Section 8 that this result fails if we replace $L \ln L$ by the related local Hardy space $h^{1}$ (see Appendix A.2). In the special case that $\left\{\Omega_{n}\right\}$ is a precompact set in $L^{1}$, and $u_{n}$ is uniformly bounded in $W^{1,2}$, this result was proved recently by $\mathrm{Li}$ and $\mathrm{Zhu}$ [1].

Remark 1.3. The compactness result rules out concentration of energy as is done in [11], i.e., concentration of $\left\|\nabla u_{n}\right\|_{L^{2}}^{2}$. In contrast, we do not rule out concentration of $\left\|\nabla u_{n}\right\|_{L^{2,1}}^{2}$ or of the corresponding second order quantity $\left\|\nabla^{2} u_{n}\right\|_{L^{1}}$. However, it will follow from our estimates (and, in particular, (6) below) that if these latter concentrations occur we must have $f_{n}$ concentrating in $L \ln L$.

Even in the classical case that $\Omega \equiv 0$ there is a consequence of such compactness which may be worth mentioning, although one which would follow from previously known theory.

Corollary 1.4. On the ball in $\mathbb{R}^{2}$, the embedding

$$
L \ln L\left(B_{1}\right) \hookrightarrow H^{-1}\left(B_{1}\right)
$$

is compact, where $H^{-1}$ is the dual space of $W_{0}^{1,2}$.

In contrast, the example given in Section 8 also serves to show that the embedding $h^{1}\left(\mathbb{R}^{2}\right) \hookrightarrow H^{-1}\left(\mathbb{R}^{2}\right)$ is not compact, which has been pointed out previously by Yuxin Ge [6, Remark 4.4]. (Strictly speaking, the example given in Section 8 and that of [6, Remark 4.4] show that the embedding $\mathcal{H}^{1}\left(\mathbb{R}^{2}\right) \hookrightarrow H^{-1}\left(\mathbb{R}^{2}\right)$ is not compact, but since $\mathcal{H}^{1}\left(\mathbb{R}^{2}\right) \hookrightarrow h^{1}\left(\mathbb{R}^{2}\right)$, this is sufficient; see Appendix A.2.)

At the heart of this paper is a collection of energy/decay estimates which we summarise in the following theorem.

Theorem 1.5 (Main supporting theorem). Suppose $u \in W^{1,2}\left(B_{1}, \mathbb{R}^{m}\right)$ is a weak solution to

$$
-\Delta u=\Omega . \nabla u+f, \quad f \in L \ln L\left(B_{1}, \mathbb{R}^{m}\right)
$$

on the unit disc in $\mathbb{R}^{2}$, where $\Omega \in L^{2}\left(B_{1}, s o(m) \otimes \mathbb{R}^{2}\right)$. Writing $\bar{u}=\frac{1}{\left|B_{1}\right|} \int_{B_{1}} u$,

1. there exist $\eta=\eta(m)>0$ and $K_{1}=K_{1}(m)<\infty$ such that if $\|\Omega\|_{L^{2}\left(B_{1}\right)} \leq \eta$, then for all $r \in(0,1 / 2]$ we have

$$
\begin{gathered}
\|\nabla u\|_{L^{2}\left(B_{r}\right)}^{2} \leq K_{1}\left(\|\Omega\|_{L^{2}\left(B_{1}\right)}^{2}\|\nabla u\|_{L^{2}\left(B_{1}\right)}^{2}+r^{2}\|u-\bar{u}\|_{L^{1}\left(B_{1}\right)}^{2}\right. \\
\left.+\|f\|_{L^{1}\left(B_{1}\right)}\|f\|_{L \ln L\left(B_{1}\right)}\right)
\end{gathered}
$$

and

(6) $\left\|\nabla^{2} u\right\|_{L^{1}\left(B_{r}\right)} \leq K_{1}\left(\|\Omega\|_{L^{2}\left(B_{1}\right)}\|\nabla u\|_{L^{2}\left(B_{1}\right)}+r^{2}\|u-\bar{u}\|_{L^{1}\left(B_{1}\right)}+\|f\|_{L \ln L\left(B_{1}\right)}\right)$;

2. for all $\delta>0$ there exist $\eta=\eta(m, \delta)>0$ and $K_{2}=K_{2}(m, \delta)<\infty$ such that if $\|\Omega\|_{L^{2}\left(B_{1}\right)} \leq \eta$, then for all $r \in(0,1]$ we have

$$
\begin{aligned}
\|\nabla u\|_{L^{2}\left(B_{r}\right)}^{2} \leq & (1+\delta) r^{2}\|\nabla u\|_{L^{2}\left(B_{1}\right)}^{2} \\
& +K_{2}\left(\|\Omega\|_{L^{2}\left(B_{1}\right)}^{2}\|\nabla u\|_{L^{2}\left(B_{1}\right)}^{2}+\|f\|_{L^{1}\left(B_{1}\right)}\|f\|_{L \ln L\left(B_{1}\right)}\right) .
\end{aligned}
$$


Although we will not need it in this work, we note that the first part of the theorem will also yield estimates for $\nabla u$ in the Lorentz space $L^{2,1}$ (by the embedding $\left.W^{1,1} \hookrightarrow L^{2,1}\right)$.

The estimates of the first part of the theorem are interior estimates which have the weakest norms of $u$ on the right-hand side. By combining them with a standard covering argument, we will also derive the following optimal global estimate:

Theorem 1.6. With $u$ and $f$ as in Theorem 1.5 and $U \Subset B_{1}$ there exist an $\eta_{1}=\eta_{1}(m)>0$ and $C=C(m, U)<\infty$ such that if $\|\Omega\|_{L^{2}\left(B_{1}\right)} \leq \eta_{1}$, then

$$
\|u\|_{W^{2,1}(U)} \leq C\left(\|u\|_{L^{1}\left(B_{1}\right)}+\|f\|_{L \ln L\left(B_{1}\right)}\right) .
$$

The second part of Theorem [1.5 is used to obtain both the regularity result (Theorem 1.1) and the compactness result (Theorem 1.2).

We remark that Theorems 1.1, 1.2, 1.5] and 1.6 all fail if we drop the antisymmetry hypothesis on $\Omega$.

The paper is laid out as follows. In Section 3 we prove the main supporting Theorem 1.5, which is central to the other results, then we go on to prove Theorem 1.6 in Section 4. This allows us to prove the compactness Theorem 1.2 in Section 5] and its corollary in Section 6. We leave the regularity Theorem 1.1 to Section 7 and finally in Section 8 we give an example to show that the compactness result fails if we replace $L \ln L$ by the local Hardy space $h^{1}$.

\section{Preliminaries}

To begin, we describe some properties of the space $L \ln L$ and record the behaviour of equations (2), (4) and various norms under scaling. In addition, we have collected a number of known results in an appendix.

2.1. Estimates for $L \ln L$. For the definition of $L \ln L$ and $f^{*}$, see Appendix A.3.

Lemma 2.1. Suppose $f \in L \ln L\left(B_{r}\left(x_{0}\right)\right)$ and $r \in(0,1 / 2]$. Then there exists $C<\infty$ such that

$$
\|f\|_{L^{1}\left(B_{r}\left(x_{0}\right)\right)} \leq C\left[\ln \left(\frac{1}{r}\right)\right]^{-1}\|f\|_{L \ln L\left(B_{r}\left(x_{0}\right)\right)} .
$$

Proof. Notice that

$$
\begin{aligned}
0 & \leq r^{2} \int_{0}^{\left|B_{1}\right|} f^{*}\left(r^{2} t\right) \ln \left(2+\frac{1}{t}\right) d t \\
& =\int_{0}^{\left|B_{r}\left(x_{0}\right)\right|} f^{*}(s) \ln \left(2+\frac{r^{2}}{s}\right) d s \\
& =\int_{0}^{\left|B_{r}\left(x_{0}\right)\right|} f^{*}(s) \ln r^{2} d s+\int_{0}^{\left|B_{r}\left(x_{0}\right)\right|} f^{*}(s) \ln \left(\frac{2}{r^{2}}+\frac{1}{s}\right) d s \\
& \leq-2 \ln \left(\frac{1}{r}\right)\|f\|_{L^{1}\left(B_{r}\left(x_{0}\right)\right)}+C\|f\|_{L \ln L\left(B_{r}\left(x_{0}\right)\right)},
\end{aligned}
$$

where the final inequality is obtained by noticing that $s \leq \pi r^{2}<1$, which implies that $\frac{2}{r^{2}}+\frac{1}{s} \leq \frac{2 \pi+1}{s} \leq\left(2+\frac{1}{s}\right)^{C}$ for some fixed $C$. 
The following lemma indicates that $L \ln L$ norms do not deteriorate under scaling. However we emphasise that they need not improve, unlike $L^{p}$ norms for $p>1$.

Lemma 2.2. Suppose $f \in L \ln L\left(B_{r}\left(x_{0}\right)\right)$, where $r \in(0,1 / 2]$. Defining $\hat{f}:=$ $r^{2} f\left(x_{0}+r x\right)$ there exists $C<\infty$ such that

$$
\|\hat{f}\|_{L \ln L\left(B_{1}\right)} \leq C\|f\|_{L \ln L\left(B_{r}\left(x_{0}\right)\right)} .
$$

Proof. First we calculate

$$
\begin{aligned}
\hat{f}^{*}(t) & =\inf \left\{s \geq 0:\left|\left\{x \in B_{1}:|\hat{f}(x)|>s\right\}\right| \leq t\right\} \\
& =\inf \left\{s \geq 0:\left|\left\{x \in B_{1}:\left|r^{2} f\left(x_{0}+r x\right)\right|>s\right\}\right| \leq t\right\} \\
& =\inf \left\{s \geq 0:\left|\left\{y \in B_{r}\left(x_{0}\right):|f(y)|>\frac{s}{r^{2}}\right\}\right| r^{-2} \leq t\right\} \\
& =r^{2} f^{*}\left(r^{2} t\right) .
\end{aligned}
$$

Therefore

$$
\begin{aligned}
\|\hat{f}\|_{L \ln L\left(B_{1}\right)} & \leq C \int_{0}^{\left|B_{1}\right|} \hat{f}^{*}(t) \ln \left(2+\frac{1}{t}\right) d t \\
& =C \int_{0}^{\left|B_{1}\right|} r^{2} f^{*}\left(r^{2} t\right) \ln \left(2+\frac{1}{t}\right) d t \\
& =C \int_{0}^{\left|B_{r}\left(x_{0}\right)\right|} f^{*}(s) \ln \left(2+\frac{r^{2}}{s}\right) d s \\
& \leq C\|f\|_{L \ln L\left(B_{r}\left(x_{0}\right)\right)}
\end{aligned}
$$

2.2. Scaling. There will be several occasions when we will require estimates on some small ball $B_{R / 2}\left(x_{0}\right)$ in terms of quantities on the ball $B_{R}\left(x_{0}\right)$. In this section, we make a note of what scaling we will be taking, and how each relevant quantity, and the equation itself, behave under this operation.

Let $u$ be a solution to (2) or (4). For $x_{0} \in B_{1}$, let $R>0$ be such that $B_{R}\left(x_{0}\right) \subset$ $B_{1}$. Now we rescale $u$ by defining $\hat{u}(x):=u\left(x_{0}+R x\right), \hat{f}(x):=R^{2} f\left(x_{0}+R x\right)$ and $\hat{\Omega}(x):=R \Omega\left(x_{0}+R x\right)$.

We have $\hat{u} \in W^{1,2}\left(B_{1}, \mathbb{R}^{m}\right)$ and

$$
\begin{aligned}
-\Delta \hat{u}(x) & =-R^{2} \Delta u\left(x_{0}+R x\right) \\
& =R \Omega\left(x_{0}+R x\right) \cdot R \nabla u\left(x_{0}+R x\right)+R^{2} f\left(x_{0}+R x\right) \\
& =\hat{\Omega}(x) \cdot \nabla \hat{u}(x)+\hat{f}(x),
\end{aligned}
$$

i.e. the same equation as before. The quantities of which we will need to keep track are:

(1) $\|\nabla \hat{u}\|_{L^{p}\left(B_{r}\right)}=R^{1-\frac{2}{p}}\|\nabla u\|_{L^{p}\left(B_{r R}\left(x_{0}\right)\right)}$ for any $r \in[0,1]$,

(2) $\|\hat{\Omega}\|_{L^{2}\left(B_{1}\right)}=\|\Omega\|_{L^{2}\left(B_{R}\left(x_{0}\right)\right)}$,

(3) $\|\hat{u}\|_{L^{p}\left(B_{1}\right)}=R^{-\frac{2}{p}}\|u\|_{L^{p}\left(B_{R}\left(x_{0}\right)\right)}$,

(4) $\|\hat{f}\|_{L^{p}\left(B_{1}\right)}=R^{2\left(1-\frac{1}{p}\right)}\|f\|_{L^{p}\left(B_{R}\left(x_{0}\right)\right)}$,

(5) $\|\hat{f}\|_{L \ln L\left(B_{1}\right)} \leq C\|f\|_{L \ln L\left(B_{R}\left(x_{0}\right)\right)}$,

where the final estimate follows from Lemma 2.2 


\section{Proof of the decay estimates, Theorem 1.5}

Most of the work in the proof will be common to both parts of the theorem. We will be referring to the $\delta$ of the second part with the understanding that in the case of the first part, we could just set $\delta=1$.

We start off with $\eta=\epsilon$, taken from Lemma A.4 and will assume throughout that $\|\Omega\|_{L^{2}\left(B_{1}\right)} \leq \eta$, with the understanding that the upper bound $\eta$ will be lowered at finitely many points during the proof. For our weak solution $u$ to (44) corresponding to $\Omega$, we will assume, without loss of generality, that $\bar{u}=\frac{1}{\left|B_{1}\right|} \int_{B_{1}} u=0$.

To begin with we use Rivière's decomposition of $\Omega$ (Lemma A.4) in order to rewrite equation (4) (equations (10) and (11) below). Lemma A.4 gives us $A \in$ $W^{1,2}\left(B_{1}, G L_{m}(\mathbb{R})\right) \cap L^{\infty}\left(B_{1}, G L_{m}(\mathbb{R})\right), B \in W^{1,2}\left(B_{1}, g l_{m}(\mathbb{R})\right)$ and $C=C(m)<\infty$ so that

$$
\nabla A-A \Omega=\nabla^{\perp} B
$$

and

$$
\|\nabla A\|_{L^{2}\left(B_{1}\right)}+\|\nabla B\|_{L^{2}\left(B_{1}\right)}+\| \operatorname{dist}\left(A, S O(m)\left\|_{L^{\infty}\left(B_{1}\right)} \leq C\right\| \Omega \|_{L^{2}\left(B_{1}\right)} .\right.
$$

Now by (8) we have

$$
\begin{aligned}
\operatorname{div}(A \nabla u) & =\nabla A . \nabla u+A \Delta u \\
& =\nabla A . \nabla u-A \Omega . \nabla u-A f \\
& =\nabla^{\perp} B . \nabla u-A f
\end{aligned}
$$

and trivially

$$
\operatorname{curl}(A \nabla u)=\nabla^{\perp} A . \nabla u .
$$

We note here that the above equations only hold in a weak sense, and more care should be taken in their calculation. We illustrate this for (10): A priori $\operatorname{div}(A \nabla u)$ is a distribution, so for $\phi \in C_{c}^{\infty}\left(B_{1}\right)$ we have

$$
\begin{aligned}
\operatorname{div}(A \nabla u)[\phi] & =-\int_{B_{1}} A \nabla u \cdot \nabla \phi \\
& =\int_{B_{1}}(\nabla A \cdot \nabla u) \phi-\nabla(\phi A) \cdot \nabla u \\
& =\int_{B_{1}}(\nabla A \cdot \nabla u) \phi-(A \Omega \cdot \nabla u) \phi-A f \phi \quad \text { since } u \text { weakly solves (4) } \\
& =\int_{B_{1}}\left(\nabla^{\perp} B \cdot \nabla u-A f\right) \phi=\left(\nabla^{\perp} B . \nabla u-A f\right)[\phi] .
\end{aligned}
$$

We will now essentially carry out a Hodge decomposition of $A \nabla u$ in $B_{1}$ using the expressions (10) and (11). We first extend all the quantities arising above to functions on $\mathbb{R}^{2}$.

Let $E x: W^{1,2}\left(B_{1}\right) \rightarrow W^{1,2}\left(\mathbb{R}^{2}\right) \cap W_{0}^{1,2}\left(B_{2}\right)$ be a bounded extension operator. Denote $\tilde{u}=E x(u) \in W^{1,2}\left(\mathbb{R}^{2}, \mathbb{R}^{m}\right)$ and note that since we are assuming $\int_{B_{1}} u=0$, by the Poincaré inequality and by standard properties of $E x$ we have

$$
\|\tilde{u}\|_{W^{1,2}\left(\mathbb{R}^{2}\right)} \leq C\|u\|_{W^{1,2}\left(B_{1}\right)} \leq C\|\nabla u\|_{L^{2}\left(B_{1}\right)}
$$

and $u=\tilde{u}$ in $B_{1}$. 
For $A$, first let $\hat{A}=A-\frac{1}{\left|B_{1}\right|} \int_{B_{1}} A$ and $\tilde{A}=E x(\hat{A}) \in W^{1,2}\left(\mathbb{R}^{2}, g l_{m}(\mathbb{R})\right)$. Noting that $\int_{B_{1}} \hat{A}=0$ and using the same argument as for $u$ we have

$$
\|\tilde{A}\|_{W^{1,2}\left(\mathbb{R}^{2}\right)} \leq C\|\nabla A\|_{L^{2}\left(B_{1}\right)} .
$$

Here we have used that $\nabla \tilde{A}=\nabla \hat{A}=\nabla A$ in $B_{1}$. Also note $\tilde{A} \nabla \tilde{u}+\left(\frac{1}{\left|B_{1}\right|} \int_{B_{1}} A\right) \nabla \tilde{u}$ $=A \nabla u$ in $B_{1}$.

We carry out the same extension for $B$ to get $\tilde{B}$ as above for $A$. We extend $f$ by zero (without relabelling), so by Appendix A.3, $f \in h^{1}\left(\mathbb{R}^{2}\right)$ with $\|f\|_{h^{1}\left(\mathbb{R}^{2}\right)} \leq$ $C\|f\|_{L \ln L\left(B_{1}\right)}$.

Now we define

$$
\begin{gathered}
D:=N\left[\nabla^{\perp} \tilde{B} \cdot \nabla \tilde{u}\right], \\
E:=N\left[\nabla^{\perp} \tilde{A} \cdot \nabla \tilde{u}\right], \\
F:=-N[A f],
\end{gathered}
$$

where $N$ is the Newtonian potential (see Appendix A.1). Note that the quantity $A f$ is well defined on the whole of $\mathbb{R}^{2}$ by the definition of $f$. Finally let

$$
H:=\tilde{A} \nabla \tilde{u}+\left(\frac{1}{\left|B_{1}\right|} \int_{B_{1}} A\right) \nabla \tilde{u}-\nabla D-\nabla F-\nabla^{\perp} E .
$$

The first thing to notice about $H$ is that

$$
H=A \nabla u-\nabla D-\nabla F-\nabla^{\perp} E
$$

in $B_{1}$. Hence we have

$$
\operatorname{div}(H)=\operatorname{div}(A \nabla u)-\Delta(D+F)=\operatorname{div}(A \nabla u)-\nabla^{\perp} \tilde{B} \cdot \nabla \tilde{u}+A f=0
$$

weakly in $B_{1}$, and a similar calculation shows $\operatorname{curl}(H)=0$ weakly in $B_{1}$. (Again care must be taken in checking these.) Therefore $H$ is harmonic in $B_{1}$ (i.e. corresponds to a harmonic 1-form).

Suppose $r \in(0,1]$. (For some estimates later it will need to be less than $\frac{1}{2}$.)

Without loss of generality, we may assume that $\delta \in(0,1]$. (Recall that when addressing the first part of the theorem, we are just setting $\delta=1$.) For $\eta$ small enough, depending on $\delta$, we may assume (by (9) that $A$ is close to a specialorthogonal matrix in the sense that both $A$ and $A^{-1}$ change the length of any vector by at most a factor of $1+\delta$. Therefore

$$
\begin{aligned}
\|\nabla u\|_{L^{2}\left(B_{r}\right)}^{2} & \leq(1+\delta)^{2}\|A \nabla u\|_{L^{2}\left(B_{r}\right)}^{2} \\
& \leq(1+3 \delta)\|A \nabla u\|_{L^{2}\left(B_{r}\right)}^{2} \\
& \leq(1+4 \delta)\|H\|_{L^{2}\left(B_{r}\right)}^{2}+C\left(\|\nabla D\|_{L^{2}\left(B_{r}\right)}^{2}+\|\nabla F\|_{L^{2}\left(B_{r}\right)}^{2}+\|\nabla E\|_{L^{2}\left(B_{r}\right)}^{2}\right),
\end{aligned}
$$

where $C$ is dependent on $\delta$. In order to obtain the inequalities of Theorem 1.5 we estimate $\|H\|_{L^{2}\left(B_{r}\right)},\|\nabla D\|_{L^{2}\left(B_{r}\right)},\|\nabla F\|_{L^{2}\left(B_{r}\right)}$ and $\|\nabla E\|_{L^{2}\left(B_{r}\right)}$.

First we consider $\nabla D=\nabla N\left[\nabla^{\perp} \tilde{B} . \nabla \tilde{u}\right]$ and $\nabla E=\nabla N\left[\nabla^{\perp} \tilde{A} . \nabla \tilde{u}\right]$. Notice that by the work of Coifman-Lions-Meyer-Semmes [2] and the fact that $\nabla N: \mathcal{H}^{1}\left(\mathbb{R}^{2}\right) \rightarrow$ 
$L^{2,1}\left(B_{1}\right)$ is a bounded linear operator (see Appendix A.4) we have

$$
\begin{aligned}
\|\nabla D\|_{L^{2}\left(B_{1}\right)}+\|\nabla E\|_{L^{2}\left(B_{1}\right)} \leq & C\left(\|\nabla D\|_{L^{2,1}\left(B_{1}\right)}+\|\nabla E\|_{L^{2,1}\left(B_{1}\right)}\right) \\
= & C\left(\left\|\nabla N\left[\nabla^{\perp} \tilde{B} \cdot \nabla \tilde{u}\right]\right\|_{L^{2,1}\left(B_{1}\right)}\right. \\
& \left.\quad+\left\|\nabla N\left[\nabla^{\perp} \tilde{A} \cdot \nabla \tilde{u}\right]\right\|_{L^{2,1}\left(B_{1}\right)}\right) \\
\leq & C\left(\left\|\nabla^{\perp} \tilde{B} \cdot \nabla \tilde{u}\right\|_{\mathcal{H}^{1}\left(\mathbb{R}^{2}\right)}+\left\|\nabla^{\perp} \tilde{A} \cdot \nabla \tilde{u}\right\|_{\mathcal{H}^{1}\left(\mathbb{R}^{2}\right)}\right) \\
\leq & C\left(\|\nabla B\|_{L^{2}\left(B_{1}\right)}+\|\nabla A\|_{L^{2}\left(B_{1}\right)}\right)\|\nabla u\|_{L^{2}\left(B_{1}\right)} \\
\leq & C\|\Omega\|_{L^{2}\left(B_{1}\right)}\|\nabla u\|_{L^{2}\left(B_{1}\right)},
\end{aligned}
$$

where we have also used the continuous embedding $L^{2,1} \hookrightarrow L^{2}$ and the estimate from Lemma A.4.

For $\nabla F=-\nabla N[A f]$ we use (Appendix A.4) that the Riesz potential $\nabla N$ : $L^{1}\left(B_{1}\right) \rightarrow L^{2, \infty}\left(B_{1}\right)$ is a bounded operator; also $\nabla N: h^{1}\left(\mathbb{R}^{2}\right) \rightarrow L^{2,1}\left(B_{1}\right)$ is bounded. We will also use the following: $L^{2, \infty}$ is the dual of $L^{2,1}$; if $f \in L \ln L\left(B_{1}\right)$, then for any $g \in L^{\infty}, g f \in L \ln L\left(B_{1}\right)$ and $\|g f\|_{L \ln L\left(B_{1}\right)} \leq\|g\|_{L^{\infty}}\|f\|_{L \ln L\left(B_{1}\right)}$ and finally we use the continuous embedding $L \ln L\left(B_{1}\right) \hookrightarrow h^{1}\left(\mathbb{R}^{2}\right)$ (see Appendix A.3). We have

$$
\begin{aligned}
\|\nabla F\|_{L^{2}\left(B_{1}\right)}^{2} & \leq C\|\nabla F\|_{L^{2, \infty}\left(B_{1}\right)}\|\nabla F\|_{L^{2,1}\left(B_{1}\right)} \\
& \leq C\|A f\|_{L^{1}\left(B_{1}\right)}\|A f\|_{h^{1}\left(\mathbb{R}^{2}\right)} \\
& \leq C\|f\|_{L^{1}\left(B_{1}\right)}\|A f\|_{L \ln L\left(B_{1}\right)} \\
& \leq C\|f\|_{L^{1}\left(B_{1}\right)}\|f\|_{L \ln L\left(B_{1}\right)} .
\end{aligned}
$$

Also, using merely the boundedness of $\nabla N: L^{1}\left(B_{1}\right) \rightarrow L^{2, \infty}\left(B_{1}\right) \hookrightarrow L^{1}\left(B_{1}\right)$ (Appendix (A.4), we have

$$
\|\nabla F\|_{L^{1}\left(B_{1}\right)} \leq C\|f\|_{L^{1}\left(B_{1}\right)} .
$$

From here, we proceed differently in order to prove the two different parts of the theorem. For the first part, we now estimate $\|H\|_{L^{1}\left(B_{2 / 3}\right)}$ and apply standard estimates for harmonic functions in order to estimate $\|H\|_{L^{2}\left(B_{r}\right)}$ : Using Lemma A.2, and estimates (14) and (16), we have

$$
\begin{aligned}
\|H\|_{L^{1}\left(B_{2 / 3}\right)} & \leq C\left(\|\nabla u\|_{L^{1}\left(B_{2 / 3}\right)}+\|\nabla D\|_{L^{1}\left(B_{2 / 3}\right)}+\|\nabla E\|_{L^{1}\left(B_{2 / 3}\right)}+\|\nabla F\|_{L^{1}\left(B_{2 / 3}\right)}\right) \\
(17) & \leq C\left(\|u\|_{L^{1}\left(B_{1}\right)}+\|f\|_{L^{1}\left(B_{1}\right)}+\|\Omega\|_{L^{2}\left(B_{1}\right)}\|\nabla u\|_{L^{2}\left(B_{1}\right)}\right) .
\end{aligned}
$$

Since $H$ is harmonic we have pointwise estimates on $H$ and its derivatives on the interior of $B_{2 / 3}$ in terms of $\|H\|_{L^{1}\left(B_{2 / 3}\right)}$, and in particular

$$
\begin{aligned}
\|H\|_{L^{\infty}\left(B_{1 / 2}\right)}+\|\nabla H\|_{\left.L^{\infty} B_{1 / 2}\right)} & \leq C\|H\|_{L^{1}\left(B_{2 / 3}\right)} \\
& \leq C\left(\|u\|_{L^{1}\left(B_{1}\right)}+\|f\|_{L^{1}\left(B_{1}\right)}+\|\Omega\|_{L^{2}\left(B_{1}\right)}\|\nabla u\|_{L^{2}\left(B_{1}\right)}\right)
\end{aligned}
$$

by (17). Therefore if we consider $r \in\left(0, \frac{1}{2}\right]$, then

$$
\begin{aligned}
\|H\|_{L^{2}\left(B_{r}\right)}^{2} & \leq \pi r^{2}\|H\|_{L^{\infty}\left(B_{r}\right)}^{2} \\
& \leq C r^{2}\left(\|u\|_{L^{1}\left(B_{1}\right)}^{2}+\|f\|_{L^{1}\left(B_{1}\right)}^{2}+\|\Omega\|_{L^{2}\left(B_{1}\right)}^{2}\|\nabla u\|_{L^{2}\left(B_{1}\right)}^{2}\right)
\end{aligned}
$$


and

$$
\begin{aligned}
\|\nabla H\|_{L^{1}\left(B_{r}\right)} & \leq \pi r^{2}\|\nabla H\|_{L^{\infty}\left(B_{r}\right)} \\
& \leq C r^{2}\left(\|u\|_{L^{1}\left(B_{1}\right)}+\|f\|_{L^{1}\left(B_{1}\right)}+\|\Omega\|_{L^{2}\left(B_{1}\right)}\|\nabla u\|_{L^{2}\left(B_{1}\right)}\right) .
\end{aligned}
$$

Now, looking back at inequality (13) and using (14), (15) and (18) we have

$$
\|\nabla u\|_{L^{2}\left(B_{r}\right)}^{2} \leq C\left(\|\Omega\|_{L^{2}\left(B_{1}\right)}^{2}\|\nabla u\|_{L^{2}\left(B_{1}\right)}^{2}+r^{2}\|u\|_{L^{1}\left(B_{1}\right)}^{2}+\|f\|_{L^{1}\left(B_{1}\right)}\|f\|_{L \ln L\left(B_{1}\right)}\right),
$$

which is the first inequality that we seek from the first part of the theorem.

In order to get the second estimate (6) of the first part of the theorem, we return to the Hodge decomposition (12), which tells us that

$$
\nabla u=A^{-1}\left(H+\nabla D+\nabla F+\nabla^{\perp} E\right)
$$

in $B_{1}$, and therefore

$$
\nabla^{2} u=\nabla A^{-1} \cdot\left(H+\nabla D+\nabla F+\nabla^{\perp} E\right)+A^{-1}\left(\nabla H+\nabla^{2} D+\nabla^{2} F+\nabla \nabla^{\perp} E\right),
$$

with

$$
\begin{aligned}
\left\|\nabla^{2} u\right\|_{L^{1}\left(B_{r}\right)} \leq & \left\|\nabla A^{-1} \cdot\left(H+\nabla D+\nabla F+\nabla^{\perp} E\right)\right\|_{L^{1}\left(B_{r}\right)} \quad(=\mathrm{I}) \\
& +\left\|A^{-1}\left(\nabla H+\nabla^{2} D+\nabla^{2} F+\nabla \nabla^{\perp} E\right)\right\|_{L^{1}\left(B_{r}\right)} . \quad(=\mathrm{II})
\end{aligned}
$$

Using (9), (14), (15) and (18) we have (also assuming $\eta<1$ )

$$
\begin{aligned}
\mathrm{I} & \leq\left\|\nabla A^{-1}\right\|_{L^{2}\left(B_{r}\right)}\left\|H+\nabla D+\nabla E+\nabla^{\perp} F\right\|_{L^{2}\left(B_{r}\right)} \\
& \leq C\left(r\|\Omega\|_{L^{2}\left(B_{1}\right)}\|u\|_{L^{1}\left(B_{1}\right)}+\|\Omega\|_{L^{2}\left(B_{1}\right)}\|\nabla u\|_{L^{2}\left(B_{1}\right)}+\|f\|_{L \ln L\left(B_{1}\right)}\right) .
\end{aligned}
$$

Now we use the fact that the operators $\nabla^{2} N: h^{1}\left(\mathbb{R}^{2}\right) \rightarrow L^{1}\left(B_{1}\right)$ and $\nabla^{2} N$ : $\mathcal{H}^{1}\left(\mathbb{R}^{2}\right) \rightarrow L^{1}\left(\mathbb{R}^{2}\right)$ are bounded (see Appendix A.2) and the estimates (9) and (19) to conclude

$$
\begin{aligned}
\mathrm{II} & \leq\left\|A^{-1}\right\|_{L^{\infty}\left(B_{r}\right)}\left\|\nabla H+\nabla^{2} D+\nabla^{2} F+\nabla \nabla^{\perp} E\right\|_{L^{1}\left(B_{r}\right)} \\
& \leq C\left(r^{2}\|u\|_{L^{1}\left(B_{1}\right)}+\|\Omega\|_{L^{2}\left(B_{1}\right)}\|\nabla u\|_{L^{2}\left(B_{1}\right)}+\|f\|_{L \ln L\left(B_{1}\right)}\right) .
\end{aligned}
$$

Since we have assumed without loss of generality that $\int_{B_{1}} u=0$, by an application of the Poincaré inequality and looking back at (20), (21) and (22) we have

$$
\left\|\nabla^{2} u\right\|_{L^{1}\left(B_{r}\right)} \leq C\left(\|\Omega\|_{L^{2}\left(B_{1}\right)}\|\nabla u\|_{L^{2}\left(B_{1}\right)}+r^{2}\|u\|_{L^{1}\left(B_{1}\right)}+\|f\|_{L \ln L\left(B_{1}\right)}\right),
$$

as desired.

For the second part of the theorem, we return to a general $r \in(0,1]$. We will now control $H$ using the standard decay estimate

$$
\|H\|_{L^{2}\left(B_{r}\right)}^{2} \leq r^{2}\|H\|_{L^{2}\left(B_{1}\right)}^{2},
$$

which holds since $|H|^{2}$ is subharmonic. 
Then using (13) and (14) and (15) again, we find that

$$
\begin{gathered}
\|\nabla u\|_{L^{2}\left(B_{r}\right)}^{2} \leq(1+4 \delta)\|H\|_{L^{2}\left(B_{r}\right)}^{2}+C\left(\|\nabla D\|_{L^{2}\left(B_{r}\right)}^{2}+\|\nabla F\|_{L^{2}\left(B_{r}\right)}^{2}+\|\nabla E\|_{L^{2}\left(B_{r}\right)}^{2}\right) \\
\leq(1+4 \delta) r^{2}\|H\|_{L^{2}\left(B_{1}\right)}^{2}+C\left(\|\nabla D\|_{L^{2}\left(B_{1}\right)}^{2}+\|\nabla F\|_{L^{2}\left(B_{1}\right)}^{2}+\|\nabla E\|_{L^{2}\left(B_{1}\right)}^{2}\right) \\
\leq(1+5 \delta) r^{2}\|A \nabla u\|_{L^{2}\left(B_{1}\right)}^{2}+C\left(\|\nabla D\|_{L^{2}\left(B_{1}\right)}^{2}+\|\nabla F\|_{L^{2}\left(B_{1}\right)}^{2}\right. \\
\left.+\|\nabla E\|_{L^{2}\left(B_{1}\right)}^{2}\right) \\
\leq(1+5 \delta)(1+\delta)^{2} r^{2}\|\nabla u\|_{L^{2}\left(B_{1}\right)}^{2}+C\left(\|\nabla D\|_{L^{2}\left(B_{1}\right)}^{2}+\|\nabla F\|_{L^{2}\left(B_{1}\right)}^{2}\right. \\
\left.+\|\nabla E\|_{L^{2}\left(B_{1}\right)}^{2}\right) \\
\leq(1+100 \delta) r^{2}\|\nabla u\|_{L^{2}\left(B_{1}\right)}^{2}+C\left(\|\Omega\|_{L^{2}\left(B_{1}\right)}^{2}\|\nabla u\|_{L^{2}\left(B_{1}\right)}^{2}\right. \\
\left.+\|f\|_{L^{1}\left(B_{1}\right)}\|f\|_{L \ln L\left(B_{1}\right)}\right) .
\end{gathered}
$$

Thus, by repeating the argument with $\delta$ reduced by a factor of 100 , we conclude the proof.

\section{Proof of the $W^{2,1}$ estimate, Theorem 1.6}

We can say immediately that the $\eta_{1}$ whose existence is claimed in the theorem can be chosen as $\eta_{1}^{2}=\min \left\{\eta^{2}, \frac{\epsilon_{0}}{K_{1}}\right\}$, where $\epsilon_{0}$ is that given in Lemma A.7 corresponding to $k=4$, and $K_{1}$ and $\eta$ are from the first part of Theorem 1.5 .

We would like to rescale the first estimate (5) of the first part of Theorem 1.5. in the case that $r=\frac{1}{2}$. Indeed, adopting the notation of Section 2.2, we know that

$$
\begin{aligned}
\|\nabla \hat{u}\|_{L^{2}\left(B_{1 / 2}\right)}^{2} & \leq K_{1}\left(\|\hat{\Omega}\|_{L^{2}\left(B_{1}\right)}^{2}\|\nabla \hat{u}\|_{L^{2}\left(B_{1}\right)}^{2}+\|\hat{u}\|_{L^{1}\left(B_{1}\right)}^{2}+\|\hat{f}\|_{L^{1}\left(B_{1}\right)}\|\hat{f}\|_{L \ln L\left(B_{1}\right)}\right) \\
& \leq K_{1}\|\hat{\Omega}\|_{L^{2}\left(B_{1}\right)}^{2}\|\nabla \hat{u}\|_{L^{2}\left(B_{1}\right)}^{2}+C\left(\|\hat{u}\|_{L^{1}\left(B_{1}\right)}^{2}+\|\hat{f}\|_{L \ln L\left(B_{1}\right)}^{2}\right)
\end{aligned}
$$

and (again by Section 2.2) this translates to

$$
\begin{aligned}
\|\nabla u\|_{L^{2}\left(B_{R / 2}\left(x_{0}\right)\right)}^{2} \leq & K_{1}\|\Omega\|_{L^{2}\left(B_{R}\left(x_{0}\right)\right)}^{2}\|\nabla u\|_{L^{2}\left(B_{R}\left(x_{0}\right)\right)}^{2} \\
& +C\left(R^{-4}\|u\|_{L^{1}\left(B_{R}\left(x_{0}\right)\right)}^{2}+\|f\|_{L \ln L\left(B_{R}\left(x_{0}\right)\right)}^{2}\right) .
\end{aligned}
$$

Using our upper bound for $\eta$ and the fact that $R \leq 1$ we have, in particular,

$$
\|\nabla u\|_{L^{2}\left(B_{R / 2}\left(x_{0}\right)\right)}^{2} \leq \epsilon_{0}\|\nabla u\|_{L^{2}\left(B_{R}\left(x_{0}\right)\right)}^{2}+C R^{-4}\left(\|u\|_{L^{1}\left(B_{1}\right)}^{2}+\|f\|_{L \ln L\left(B_{1}\right)}^{2}\right) .
$$

Letting $\Gamma=C\left(\|u\|_{L^{1}\left(B_{1}\right)}^{2}+\|f\|_{L \ln L\left(B_{1}\right)}^{2}\right)$ we are precisely in the setup of Lemma A.7. since this estimate is true in particular for all $B_{2 R}\left(x_{0}\right) \subset B_{1}$. Therefore

$$
\|\nabla u\|_{L^{2}\left(B_{1 / 2}\right)} \leq C\left(\|u\|_{L^{1}\left(B_{1}\right)}+\|f\|_{L \ln L\left(B_{1}\right)}\right) .
$$

It remains to improve this estimate to control the second derivatives, and for that we use the second estimate (6) of the first part of Theorem [1.5, in the case $r=\frac{1}{2}$, which we then scale by a factor $\frac{1}{2}$ to give:

$$
\left\|\nabla^{2} u\right\|_{L^{1}\left(B_{1 / 4}\right)} \leq C\left(\|\Omega\|_{L^{2}\left(B_{1 / 2}\right)}\|\nabla u\|_{L^{2}\left(B_{1 / 2}\right)}+\|u\|_{L^{1}\left(B_{1 / 2}\right)}+\|f\|_{L \ln L\left(B_{1 / 2}\right)}\right) .
$$

Combining with (23) then yields

$$
\left\|\nabla^{2} u\right\|_{L^{1}\left(B_{1 / 4}\right)} \leq C\left(\|u\|_{L^{1}\left(B_{1}\right)}+\|f\|_{L \ln L\left(B_{1}\right)}\right),
$$


and a simple rescaling and covering argument gives us that for any compactly contained $U \Subset B_{1}$ there is a $C=C(U, m)<\infty$ such that

$$
\|u\|_{W^{2,1}(U)} \leq C\left(\|u\|_{L^{1}\left(B_{1}\right)}+\|f\|_{L \ln L\left(B_{1}\right)}\right) .
$$

\section{Proof of the compactness, Theorem 1.2}

Here we pick $\eta_{2}=\min \left\{\eta_{1}, \eta, \sqrt{\frac{1}{2 K_{2}}}\right\}$, where $\eta_{1}$ is from Theorem 1.6, and $\eta$ and $K_{2}$ are from the second part of Theorem 1.5 for the choice $\delta=1$. We know (by Theorem (1.6) that for all $U \Subset B_{1}$, our sequence $\left\{u_{n}\right\}$ is uniformly bounded in $W^{2,1}(U)$, so by the Sobolev embedding theorem there exists some $u \in W_{l o c}^{1,2}\left(B_{1}\right)$ such that (up to a subsequence) $u_{n} \rightarrow u$ weakly in $W^{1,2}\left(B_{2 / 3}\right)$. We also know that $\left\{\nabla u_{n}\right\}$ is uniformly bounded in $W^{1,1}\left(B_{2 / 3}\right)$, so by Lemma A.6 (with $\nabla u_{n}=V_{n}$ ) if we have

$$
\lim _{r \downarrow 0} \limsup _{n \rightarrow \infty}\left\|\nabla u_{n}\right\|_{L^{2}\left(B_{r}(x)\right)}=0
$$

for all $x \in B_{2 / 3}$, then

$$
\nabla u_{n} \rightarrow \nabla u
$$

strongly in $L_{l o c}^{2}\left(B_{2 / 3}\right)$, which would prove the theorem. Therefore, it remains to prove (24).

Now pick $x_{0} \in B_{2 / 3}$ and $R \in(0,1 / 2]$ small enough such that $B_{R}\left(x_{0}\right) \subset B_{2 / 3}$. Applying the second part of Theorem 1.5 to the rescaled scenario from Section 2.2 (for each $n$ ) yields (for $r \in(0,1]$ )

$$
\begin{aligned}
\left\|\nabla \hat{u}_{n}\right\|_{L^{2}\left(B_{r}\right)}^{2} \leq & K_{2}\left\|\hat{\Omega}_{n}\right\|_{L^{2}\left(B_{1}\right)}^{2}\left\|\nabla \hat{u}_{n}\right\|_{L^{2}\left(B_{1}\right)}^{2}+2 r^{2}\left\|\nabla \hat{u}_{n}\right\|_{L^{2}\left(B_{1}\right)}^{2} \\
& +K_{2}\left\|\hat{f}_{n}\right\|_{L^{1}\left(B_{1}\right)}\left\|\hat{f}_{n}\right\|_{L \ln L\left(B_{1}\right)}
\end{aligned}
$$

and reversing the scaling leaves us with

$$
\begin{aligned}
\left\|\nabla u_{n}\right\|_{L^{2}\left(B_{r R}\left(x_{0}\right)\right)}^{2} \leq & K_{2}\left\|\Omega_{n}\right\|_{L^{2}\left(B_{R}\left(x_{0}\right)\right)}^{2}\left\|\nabla u_{n}\right\|_{L^{2}\left(B_{R}\left(x_{0}\right)\right)}^{2} \\
& +2 r^{2}\left\|\nabla u_{n}\right\|_{L^{2}\left(B_{R}\left(x_{0}\right)\right)}^{2}+K_{2}\left\|f_{n}\right\|_{L^{1}\left(B_{R}\left(x_{0}\right)\right)}\left\|f_{n}\right\|_{L \ln L\left(B_{R}\left(x_{0}\right)\right)} \\
\leq & \frac{1}{2}\left\|\nabla u_{n}\right\|_{L^{2}\left(B_{R}\left(x_{0}\right)\right)}^{2} \\
& +C\left(r^{2}\left\|\nabla u_{n}\right\|_{L^{2}\left(B_{R}\left(x_{0}\right)\right)}^{2}+\left[\ln \left(\frac{1}{R}\right)\right]^{-1}\left\|f_{n}\right\|_{L \ln L\left(B_{R}\left(x_{0}\right)\right)}^{2}\right)
\end{aligned}
$$

using Lemma 2.1 .

Now, using that $\left\{u_{n}\right\}$ is uniformly bounded in $W^{2,1}\left(B_{2 / 3}\right)$ and the hypotheses of the theorem, we have

$$
\left\|\nabla u_{n}\right\|_{L^{2}\left(B_{r R}\left(x_{0}\right)\right)}^{2} \leq(1 / 2)\left\|\nabla u_{n}\right\|_{L^{2}\left(B_{R}\left(x_{0}\right)\right)}^{2}+C\left(r^{2}+\left[\ln \left(\frac{1}{R}\right)\right]^{-1}\right) .
$$

Hence

$$
\lim _{R \downarrow 0} \lim _{r \downarrow 0} \limsup _{n \rightarrow \infty}\left\|\nabla u_{n}\right\|_{L^{2}\left(B_{r R}\left(x_{0}\right)\right)}^{2} \leq(1 / 2) \lim _{R \downarrow 0} \lim _{r \downarrow 0} \limsup _{n \rightarrow \infty}\left\|\nabla u_{n}\right\|_{L^{2}\left(B_{R}\left(x_{0}\right)\right)}^{2}
$$

and we have shown that

$$
\lim _{r \downarrow 0} \limsup _{n \rightarrow \infty}\left\|\nabla u_{n}\right\|_{L^{2}\left(B_{r}\left(x_{0}\right)\right)}=0,
$$

which proves the theorem. 
6. Proof of the compact embedding $L \ln L \hookrightarrow H^{-1}$, Corollary 1.4

In this section we use $\mathcal{H}^{1}-B M O$ duality (Appendix A.2 and [5]), the compactness result (Theorem 1.2) and the continuous embedding $W^{1,2}\left(\mathbb{R}^{2}\right) \hookrightarrow B M O\left(\mathbb{R}^{2}\right)$ (Appendix A.4) to prove the compactness of the embedding $L \ln L\left(B_{1}\right) \hookrightarrow H^{-1}\left(B_{1}\right)$.

First we check that the embedding $L \ln L \hookrightarrow H^{-1}$ exists and is continuous. We will realise $f \in L \ln L\left(B_{1}\right)$ as a bounded linear functional on $W_{0}^{1,2}\left(B_{1}\right)$.

Recall from Appendix A.3 that if $f \in L \ln L\left(B_{1}\right)$, then $f-\bar{f} \in \mathcal{H}^{1}\left(\mathbb{R}^{2}\right)$ and $\|f-\bar{f}\|_{\mathcal{H}^{1}\left(\mathbb{R}^{2}\right)} \leq C\|f\|_{L \ln L\left(B_{1}\right)}$. For $\phi \in W_{0}^{1,2}\left(B_{1}\right)$ we extend it by zero and calculate

$$
\begin{aligned}
\int_{B_{1}} f \phi & =\int(f-\bar{f}) \phi+\int \bar{f} \phi \\
& \leq C\|f-\bar{f}\|_{\mathcal{H}^{1}\left(\mathbb{R}^{m}\right)}\|\phi\|_{B M O\left(\mathbb{R}^{2}\right)}+\frac{1}{\pi}\|f\|_{L^{1}\left(B_{1}\right)}\|\phi\|_{L^{1}\left(B_{1}\right)} \\
& \leq C\|f\|_{L \ln L\left(B_{1}\right)}\|\phi\|_{W_{0}^{1,2}\left(B_{1}\right)} .
\end{aligned}
$$

Thus $f \in H^{-1}\left(B_{1}\right)$ and $\|f\|_{H^{-1}\left(B_{1}\right)} \leq C\|f\|_{L \ln L\left(B_{1}\right)}$.

Now consider a sequence $\left\{f_{n}\right\} \subset L \ln L\left(B_{1}\right)$ such that $\left\|f_{n}\right\|_{L \ln L\left(B_{1}\right)} \leq \Lambda<\infty$. We can extend each $f_{n}$ to be zero outside $B_{1}$ and consider the sequence of solutions $\left\{u_{n}\right\} \subset W_{0}^{1,2}\left(B_{2}\right)$ weakly solving

$$
-\Delta u_{n}=f_{n} \quad \text { on } B_{2} .
$$

By the compactness of Theorem 1.2 for $\Omega_{n} \equiv 0$ we can conclude that there exists some $u \in W^{1,2}\left(B_{2}\right)$ such that (up to a subsequence) $u_{n} \rightarrow u$ strongly in $W^{1,2}\left(B_{1}\right)$.

Writing $f=-\Delta u$ (which can clearly be viewed as an element of $H^{-1}\left(B_{1}\right)$ ) we see that

$$
\begin{aligned}
\left\|f_{n}-f\right\|_{H^{-1}\left(B_{1}\right)} & =\sup _{\phi \in W_{0}^{1,2}\left(B_{1}\right)\|\phi\|_{W_{0}^{1,2}\left(B_{1}\right)}=1} \int\left(f_{n}-f\right) \phi \\
& =\sup _{\phi \in W_{0}^{1,2}\left(B_{1}\right)\|\phi\|_{W_{0}^{1,2}\left(B_{1}\right)}=1} \int\left(\nabla u_{n}-\nabla u\right) \cdot \nabla \phi \\
& \leq\left\|\nabla u_{n}-\nabla u\right\|_{L^{2}\left(B_{1}\right)} \rightarrow 0
\end{aligned}
$$

as $n \rightarrow \infty$.

\section{Proof of the optimal Regularity, Theorem 1.1}

The proof will proceed broadly in two steps. First, we will use a type of 'geometric bootstrapping' to show that solutions $u$ have almost the optimal regularity claimed.

Lemma 7.1. Suppose $u \in W^{1,2}\left(B_{1}, \mathbb{R}^{m}\right)$ is a weak solution to

$$
-\Delta u=\Omega . \nabla u+f,
$$

where $\Omega \in L^{2}\left(B_{1}\right.$, so $\left.(m) \otimes \mathbb{R}^{2}\right)$ and $f \in L^{p}$ for some $p \in(1,2)$. Then $u \in W_{l o c}^{2, \gamma}\left(B_{1}\right)$ for all $\gamma \in[1, p)$.

Once we have got this far, we can quantify the $W^{2, \gamma}$ regularity via the following estimate. 
Lemma 7.2. Let $p \in(1,2)$ and suppose $\gamma \in\left(\frac{p+1}{2}, p\right)$ and $u \in W^{2, \gamma}\left(B_{1}, \mathbb{R}^{m}\right)$ solves

$$
-\Delta u=\Omega . \nabla u+f,
$$

where $\Omega \in L^{2}\left(B_{1}, s o(m) \otimes \mathbb{R}^{2}\right)$ and $f \in L^{p}$. Then there exists $\eta_{3}=\eta_{3}(p, m)>0$ and $C=C(p, m)<\infty$ such that if $\|\Omega\|_{L^{2}\left(B_{1}\right)} \leq \eta_{3}$, then

$$
\|u\|_{W^{2, \gamma}\left(B_{1 / 2}\right)} \leq C\left(\|f\|_{L^{p}\left(B_{1}\right)}+\|u\|_{L^{1}\left(B_{1}\right)}\right) .
$$

Let us assume these two lemmata for the moment and see how Theorem 1.1 follows. First, Lemma 7.1 directly applies, and we deduce $W_{l o c}^{2, \gamma}\left(B_{1}\right)$ regularity for $\gamma \in[1, p)$. If we assume that $\|\Omega\|_{L^{2}\left(B_{1}\right)} \leq \eta_{3}$, then (25) of Lemma 7.2 holds for $\gamma \in\left(\frac{p+1}{2}, p\right)$ (strictly speaking we should make a small rescaling so that we can assume that $u \in W^{2, \gamma}\left(B_{1}\right)$ for $\left.\gamma \in[1, p)\right)$ and by taking the limit $\gamma \nearrow p$, we deduce that

$$
\|u\|_{W^{2, p}\left(B_{1 / 2}\right)} \leq C\left(\|f\|_{L^{p}\left(B_{1}\right)}+\|u\|_{L^{1}\left(B_{1}\right)}\right) .
$$

By an appropriate covering argument, working on balls small enough so that $\|\Omega\|_{L^{2}} \leq \eta_{3}$, we deduce the regularity claimed in the theorem, and the claimed estimate (3).

It remains therefore to prove Lemmata 7.1 and 7.2 . We will need, in turn, an additional lemma, which expresses the decay of energy of solutions $u$.

Lemma 7.3. Under the hypotheses of Lemma 7.1, set $\alpha=2(1-1 / p) \in(0,1)$. Then there exists $\eta_{4}>0$ depending on $p$ and $m$ such that if $\|\Omega\|_{L^{2}\left(B_{1}\right)} \leq \eta_{4}$, then

$$
\sup _{x_{0} \in B_{1 / 2}, r \in(0,1 / 2)} r^{-2 \alpha}\|\nabla u\|_{L^{2}\left(B_{r}\right)}^{2}<\infty
$$

and, in particular, so that

$$
\sup _{x_{0} \in B_{1 / 2}, r \in(0,1 / 2)} r^{-\alpha}\|\Omega . \nabla u\|_{L^{1}\left(B_{r}\right)}<\infty .
$$

Proof of Lemma 7.3. For reasons that will become apparent, choose $\delta \in(0,1]$ sufficiently small so that

$$
\lambda:=\frac{(1+2 \delta)}{4}<2^{-4(1-1 / p)}=: \Lambda \in\left(\frac{1}{4}, 1\right) .
$$

We can now choose $\eta_{4}:=\min \left\{\eta, \sqrt{\frac{\delta}{4 K_{2}}}\right\}$, where $\eta$ is from the second part of Theorem 1.5, depending on the $\delta$ we have just chosen and therefore on $p$ (as well as $m$ ) and where $K_{2}$ is also from the second part of Theorem 1.5. Now take an arbitrary point $x_{0} \in B_{1 / 2}$ and any $R \in(0,1 / 2)$. Estimate (77) in the case that $r=\frac{1}{2}$, applied to the rescaled quantities defined in Section 2.2 yields

$$
\begin{aligned}
\|\nabla \hat{u}\|_{L^{2}\left(B_{1 / 2}\right)}^{2} \leq & \frac{(1+\delta)}{4}\|\nabla \hat{u}\|_{L^{2}\left(B_{1}\right)}^{2} \\
& +K_{2}\left(\|\hat{\Omega}\|_{L^{2}\left(B_{1}\right)}^{2}\|\nabla \hat{u}\|_{L^{2}\left(B_{1}\right)}^{2}+\|\hat{f}\|_{L^{1}\left(B_{1}\right)}\|\hat{f}\|_{L \ln L\left(B_{1}\right)}\right) \\
\leq & \lambda\|\nabla \hat{u}\|_{L^{2}\left(B_{1}\right)}^{2}+C\|\hat{f}\|_{L^{p}\left(B_{1}\right)}^{2} .
\end{aligned}
$$

Reversing the scaling, using Section 2.2 , we find that

$$
\begin{aligned}
\|\nabla u\|_{L^{2}\left(B_{R / 2}\left(x_{0}\right)\right)}^{2} & \leq \lambda\|\nabla u\|_{L^{2}\left(B_{R}\left(x_{0}\right)\right)}^{2}+C R^{4(1-1 / p)}\|f\|_{L^{p}\left(B_{R}\left(x_{0}\right)\right)}^{2} \\
& \leq \lambda\|\nabla u\|_{L^{2}\left(B_{R}\left(x_{0}\right)\right)}^{2}+\left[C\|f\|_{L^{p}\left(B_{1}\right)}^{2}\right] R^{4(1-1 / p)} .
\end{aligned}
$$


Now applying what we have proved for $R=2^{-k}$, with $k \in\{1,2, \ldots\}$ and using the abbreviation $a_{k}:=\|\nabla u\|_{L^{2}\left(B_{2}-k\right.}^{\left.2\left(x_{0}\right)\right)}$, we find that

$$
\begin{aligned}
a_{k+1} & \leq \lambda a_{k}+K_{3} 2^{-4(1-1 / p) k} \\
& =\lambda a_{k}+K_{3} \Lambda^{k},
\end{aligned}
$$

where $K_{3}$ is independent of $x_{0}$. This recursion relation can be solved to yield

$$
a_{k+1} \leq \lambda^{k} a_{1}+K_{3} \Lambda \frac{\left(\Lambda^{k}-\lambda^{k}\right)}{\Lambda-\lambda}
$$

and by (26), this simplifies to

$$
\|\nabla u\|_{L^{2}\left(B_{2-k}\left(x_{0}\right)\right)}^{2}=: a_{k} \leq C \Lambda^{k} .
$$

Thus, for $r \in(0,1 / 2]$ we have

$$
\|\nabla u\|_{L^{2}\left(B_{r}\left(x_{0}\right)\right)}^{2} \leq C r^{4(1-1 / p)},
$$

and hence the lemma is proved.

Proof of Lemma 7.1. Our goal is to prove $W^{2, \gamma}$ regularity, for all $\gamma \in[1, p)$. By applying Calderón-Zygmund theory directly to equation (2), this will follow if we can show that $\Omega . \nabla u \in L^{s}$ for all $s \in[1, p)$.

By the nature of what we are trying to prove, we may also assume that $\|\Omega\|_{L^{2}\left(B_{1}\right)}$ $\leq \eta_{4}$, where $\eta_{4}$ is from Lemma 7.3 . If this were not true, we would apply the result, after rescaling, on appropriate small balls where it is true.

By Lemma 7.3, and the first part of Lemma A.3 with $h$ chosen to be $\Omega . \nabla u$, we can deduce that for any $q \in\left(1, \frac{2-\alpha}{1-\alpha}\right)=\left(1, \frac{2}{2-p}\right)$, we have

$$
I_{1} h \in L^{q}\left(B_{1 / 4}\right),
$$

and, in particular, this implies that $u \in W^{1, q}\left(B_{1 / 4}\right)$ for some $q>2$. If we apply the same result over appropriate smaller balls, we have in fact that $u \in W_{l o c}^{1, q}\left(B_{1}\right)$ for some $q>2$. At that point, we know that $\Omega$. $\nabla u \in L_{l o c}^{s}\left(B_{1}\right)$ with $s=\frac{2 q}{2+q} \in$ $\left(1, \frac{2}{3-p}\right) \subset(1, p)$.

We will now show that from here it is possible to carry out a geometric bootstrapping argument by using the second part of Lemma A.3. As an aside, we note that a bootstrapping argument using only classical Calderón-Zygmund methods does not work. The bootstrapping claim is that

$$
\Omega . \nabla u \in L_{l o c}^{s}\left(B_{1}\right) \text { with } s \in(1, p) \Longrightarrow \Omega . \nabla u \in L_{l o c}^{s\left(\frac{2}{2+s-p}\right)}\left(B_{1}\right) .
$$

This is true because whenever we know that $\Omega . \nabla u \in L_{l o c}^{s}\left(B_{1}\right)$ with $s \in(1, p)$, then we can deduce from Lemma 7.3 and the second part of Lemma A.3 with $h$ chosen to be $\Omega . \nabla u$, that

$$
I_{1} h \in L^{s \frac{2-\alpha}{1-\alpha}}\left(B_{1 / 4}\right),
$$

and recalling that $\frac{2-\alpha}{1-\alpha}=\frac{2}{2-p}$, this is enough to establish this time that $u \in$ $W^{1, \frac{2 s}{2-p}}\left(B_{1 / 4}\right)$ and hence $\Omega . \nabla u \in L^{s\left(\frac{2}{2+s-p}\right)}\left(B_{1 / 4}\right)$. Again, by a simple covering argument, we deduce that $\Omega . \nabla u \in L_{l o c}^{s\left(\frac{2}{2+s-p}\right)}\left(B_{1}\right)$ as desired.

By iterating the bootstrapping claim (27), we find that $\Omega . \nabla u \in L_{l o c}^{s}\left(B_{1}\right)$ for all $s \in[1, p)$, and the proof is complete. 
It remains to prove Lemma 7.2

Proof of Lemma 7.2. We begin by applying the Calderón-Zygmund estimate from Lemma A.1 giving some $C$ independent of $\gamma$ such that

$$
\|u\|_{W^{2, \gamma}\left(B_{1 / 2}\right)} \leq \frac{C}{\gamma-1}\left(\|\Delta u\|_{L^{\gamma}\left(B_{2 / 3}\right)}+\|u\|_{L^{\gamma}\left(B_{2 / 3}\right)}\right),
$$

valid for $\gamma \in(1,2]$ and $u \in W^{2, \gamma}\left(B_{1}\right)$.

For the specific $u$ of the lemma, and $\gamma \in\left(\frac{1+p}{2}, p\right)$, we may then compute (also using the inequalities of Sobolev and Hölder, and Lemma A.2

$$
\begin{aligned}
\|u\|_{W^{2, \gamma}\left(B_{1 / 2}\right)} & \leq C\left(\|\Omega . \nabla u\|_{L^{\gamma}\left(B_{2 / 3}\right)}+\|f\|_{L^{\gamma}\left(B_{2 / 3}\right)}+\|u\|_{L^{2}\left(B_{2 / 3}\right)}\right) \\
& \leq C\left(\|\Omega . \nabla u\|_{L^{\gamma}\left(B_{2 / 3}\right)}+\|f\|_{L^{\gamma}\left(B_{2 / 3}\right)}+\|u\|_{W^{1,1}\left(B_{2 / 3}\right)}\right) \\
& \leq C\left(\|\Omega\|_{L^{2}\left(B_{1}\right)}\|\nabla u\|_{L^{\frac{2 \gamma}{2-\gamma}\left(B_{1}\right)}}+\|f\|_{L^{\gamma}\left(B_{1}\right)}+\|u\|_{L^{1}\left(B_{1}\right)}\right),
\end{aligned}
$$

where $C$ depends only on $p$ and $m$. Now consider any $v \in W^{2, t}\left(B_{1 / 2}\right)$ for $t \in[1,2)$. We can find a $t$-independent extension operator $E x: W^{2, t}\left(B_{1 / 2}\right) \rightarrow W^{2, t}\left(\mathbb{R}^{2}\right)$ whose images have compact support in $B_{1}$, so that there exists some $C<\infty$ independent of $t \in[1,2)$ such that (denoting $E x(v)=\tilde{v}$ )

$$
\|\tilde{v}\|_{W^{2, t}\left(\mathbb{R}^{2}\right)} \leq C\|v\|_{W^{2, t}\left(B_{1 / 2}\right)}
$$

with $v=\tilde{v}$ in $B_{1 / 2}$. (See for instance [7, Theorem 7.25].)

From here we apply the standard Sobolev embedding for $\nabla \tilde{v} \in W^{1, t} \hookrightarrow L^{\frac{2 t}{2-t}}$ to obtain (see [7, Theorem 7.10])

$$
\|\nabla \tilde{v}\|_{L^{\frac{2 t}{2-t}}} \leq \frac{t}{2-t}\left\|\nabla^{2} \tilde{v}\right\|_{L^{t}}
$$

This, coupled with (29), gives us

$$
\|\nabla v\|_{L^{\frac{2 t}{2-t}\left(B_{1 / 2}\right)}} \leq \frac{C}{2-t}\|v\|_{W^{2, t}\left(B_{1 / 2}\right)}
$$

for all $v \in W^{2, t}\left(B_{1 / 2}\right), t \in[1,2)$ and where $C$ is independent of $t$.

Using (28) and (30) we have that there exists some $C<\infty$ depending only on $p$ and $m$ such that

$$
\|\nabla u\|_{L^{\frac{2 \gamma}{2-\gamma}\left(B_{1 / 2}\right)}} \leq C\left(\|\Omega\|_{L^{2}\left(B_{1}\right)}\|\nabla u\|_{L^{\frac{2 \gamma}{2-\gamma}\left(B_{1}\right)}}+\|f\|_{L^{\gamma}\left(B_{1}\right)}+\|u\|_{L^{1}\left(B_{1}\right)}\right)
$$

for all $\gamma \in\left(\frac{1+p}{2}, p\right)$.

We now choose $B_{R}\left(x_{0}\right) \subset B_{1}$; using the scaling of Section 2.2 estimate (31) yields

$$
\|\nabla \hat{u}\|_{L^{\frac{2 \gamma}{2-\gamma}\left(B_{1 / 2}\right)}} \leq C\left(\|\hat{\Omega}\|_{L^{2}\left(B_{1}\right)}\|\nabla \hat{u}\|_{L^{\frac{2 \gamma}{2-\gamma}\left(B_{1}\right)}}+\|\hat{f}\|_{L^{\gamma}\left(B_{1}\right)}+\|\hat{u}\|_{L^{1}\left(B_{1}\right)}\right),
$$

which translates (using $R \leq 1$ and Section 2.2) to

$$
\begin{aligned}
\|\nabla u\|_{L^{\frac{2 \gamma}{2-\gamma}\left(B_{R / 2}\left(x_{0}\right)\right)}} \leq C( & \|\Omega\|_{L^{2}\left(B_{R}\left(x_{0}\right)\right)}\|\nabla u\|_{L^{\frac{2 \gamma}{2-\gamma}}\left(B_{R}\left(x_{0}\right)\right)} \\
& \left.+R^{-3}\left(\|f\|_{L^{\gamma}\left(B_{R}\left(x_{0}\right)\right)}+\|u\|_{L^{1}\left(B_{R}\left(x_{0}\right)\right)}\right)\right) .
\end{aligned}
$$


Now let $\beta=\frac{2 \gamma}{2-\gamma}$ and raise this inequality to the power $\beta$ to obtain (noticing $\left.\beta<\frac{2 p}{2-p}\right)$

$$
\begin{aligned}
\|\nabla u\|_{L^{\beta}\left(B_{R / 2}\left(x_{0}\right)\right)}^{\beta} \leq & K\|\Omega\|_{L^{2}\left(B_{R}\left(x_{0}\right)\right)}^{\beta}\|\nabla u\|_{L^{\beta}\left(B_{R}\left(x_{0}\right)\right)}^{\beta} \\
& +R^{-\frac{6 p}{2-p}} K\left(\|f\|_{L^{\gamma}\left(B_{R}\left(x_{0}\right)\right)}+\|u\|_{L^{1}\left(B_{R}\left(x_{0}\right)\right)}\right)^{\beta}
\end{aligned}
$$

for some specific $K<\infty$ depending only on $p$ and $m$.

We now wish to apply Lemma A.7. We are able to choose $\eta_{3}=\left(\frac{\epsilon_{0}}{K}\right)^{\frac{1}{\beta}}$, where $\epsilon_{0}$ is that of Lemma A.7 corresponding to the choice $k=\frac{6 p}{2-p}$. Let $\Gamma=K\left(\|f\|_{L^{\gamma}\left(B_{1}\right)}+\right.$ $\left.\|u\|_{L^{1}\left(B_{1}\right)}\right)^{\beta}$.

The above estimate holds in particular for any $B_{R}\left(x_{0}\right)$ such that $B_{2 R}\left(x_{0}\right) \subset B_{1}$, so we have

$$
\|\nabla u\|_{L^{\beta}\left(B_{R / 2}\left(x_{0}\right)\right)}^{\beta} \leq \epsilon_{0}\|\nabla u\|_{L^{\beta}\left(B_{R}\left(x_{0}\right)\right)}^{\beta}+R^{-\frac{6 p}{2-p}} \Gamma .
$$

Hence a direct application of Lemma A.7 gives us

$$
\|\nabla u\|_{L^{\frac{2 \gamma}{2-\gamma}\left(B_{1 / 2}\right)}} \leq C\left(\|f\|_{L^{p}\left(B_{1}\right)}+\|u\|_{L^{1}\left(B_{1}\right)}\right) .
$$

Therefore a simple covering argument yields that there is a $C=C(p, m)<\infty$ such that

$$
\|\nabla u\|_{L^{\frac{2 \gamma}{2-\gamma}\left(B_{2 / 3}\right)}} \leq C\left(\|f\|_{L^{p}\left(B_{1}\right)}+\|u\|_{L^{1}\left(B_{1}\right)}\right),
$$

and from here another application of Lemmata A.1 and A.2 tells us that there is a $C=C(p, m)<\infty$ such that

$$
\|u\|_{W^{2, \gamma}\left(B_{1 / 2}\right)} \leq C\left(\|f\|_{L^{p}\left(B_{1}\right)}+\|u\|_{L^{1}\left(B_{1}\right)}\right) .
$$

\section{8. $L \ln L$ CANNOT BE REPLACED BY $h^{1}$}

Here we present a counterexample to the compactness Theorem 1.2 when we allow $f_{n} \in h^{1}\left(B_{1}\right)$. Our example will have $\Omega_{n} \equiv 0$ for all $n$ and $u_{n}: B_{1} \rightarrow \mathbb{S}^{2}$ will be a sequence of harmonic maps with bounded energy that undergoes bubbling.

Let $\pi: \mathbb{R}^{2} \rightarrow \mathbb{S}^{2}$ be the (inverse of) stereographic projection and take $u_{n}(x, y)=$ $\pi(n x, n y)$. Since $u_{n}$ is harmonic for all $n$ we know it solves (see [9])

$$
-\Delta u_{n}=\nabla^{\perp} B_{n} . \nabla u_{n},
$$

where $\nabla^{\perp}\left(B_{n}\right)_{j}^{i}=\left(u_{n}\right)^{i} \nabla\left(u_{n}\right)^{j}-\left(u_{n}\right)^{j} \nabla\left(u_{n}\right)^{i} ;$ therefore $\left\|\nabla^{\perp} B_{n}\right\|_{L^{2}\left(\mathbb{R}^{2}\right)} \leq$ $C\left\|\nabla u_{n}\right\|_{L^{2}\left(\mathbb{R}^{2}\right)}$. Letting $f_{n}=\nabla^{\perp} B_{n}$. $\nabla u_{n}$ we have

$$
\left\|f_{n}\right\|_{\mathcal{H}^{1}\left(\mathbb{R}^{2}\right)} \leq C\left\|\nabla u_{n}\right\|_{L^{2}\left(\mathbb{R}^{2}\right)}^{2} \leq C\|\nabla \pi\|_{L^{2}\left(\mathbb{R}^{2}\right)}^{2}=\Lambda<\infty .
$$

This tells us two things: first that $\left\|f_{n}\right\|_{h^{1}\left(B_{1}\right)} \leq \Lambda<\infty$ and second that $\left\|u_{n}\right\|_{W^{1,2}\left(B_{1}\right)}$ $\leq \Lambda<\infty$. At this point we have all the hypotheses of the theorem (except that we allow $f_{n} \in h^{1}$ ), but it is easy to see that there can be no subsequence converging locally strongly in $W^{1,2}$ because this sequence forms a bubble at the origin. 


\section{Appendix A. BACKGROUnd AND SUPPORTING RESUlts}

A.1. Singular integrals. We recall here the basics of Calderón-Zygmund theory on the unit ball $B_{1} \subset \mathbb{R}^{2}$, following 7 . Define the Newtonian potential operator $N$ on functions $f \in L^{1}\left(B_{1}\right)$ (implicitly extended to be zero to $\mathbb{R}^{2} \backslash B_{1}$ ) by

$$
N[f](x):=(\Gamma * f)(x)=\int \Gamma(x-y) f(y) d y,
$$

where $\Gamma(x)=\frac{1}{2 \pi} \ln |x|$. If $f \in C_{c}^{\infty}$, then $\Delta N[f]=f$. Writing $w=N[f]$ we have

(1) $N: L^{p}\left(B_{1}\right) \rightarrow L^{p}\left(B_{1}\right)$ is a bounded operator for all $1 \leq p \leq \infty$.

(2) $\nabla w=\nabla N[f]=(\nabla \Gamma) * f$. We will frequently view $\nabla N$ as an operator in its own right. It is easy to see that $|\nabla N[f]| \leq \frac{1}{2 \pi} I_{1}[|f|]$, where $I_{1}$ is the standard notation for this Riesz potential (defined by convolution with $\frac{1}{|x|}$ ) and $I_{1}: L^{p}\left(B_{1}\right) \rightarrow L^{\frac{2 p}{2-p}}\left(B_{1}\right)$ is a bounded operator for all $1<p<2$.

(3) (Calderón-Zygmund) Let $f \in L^{p}\left(B_{1}\right), 1<p<\infty$ and $w=N[f]$. Then $\nabla^{2} w=\nabla^{2} N[f]=\left(\nabla^{2} \Gamma\right) * f$ and (as above we see $\nabla^{2} N$ as an operator) $\nabla^{2} N: L^{p}\left(B_{1}\right) \rightarrow L^{p}\left(B_{1}\right)$ is bounded for $p$ in this range. More explicitly we have $w \in W^{2, p}\left(B_{1}\right), \Delta w=f$ almost everywhere, and

$$
\left\|\nabla^{2} w\right\|_{L^{p}\left(B_{1}\right)} \leq C(p)\|f\|_{L^{p}\left(B_{1}\right)} .
$$

In fact, revisiting a second time the proof of the Calderón-Zygmund estimates (e.g. [7. §9.4], but interpolating between $q=1$ and $r=3$ ) we find that the dependency of $C$ in (32) can be weakened, and one can prove:

Lemma A.1. Let $\gamma \in(1,2]$ and suppose $u \in W^{2, \gamma}\left(B_{1}\right)$. Then there exists some $C<\infty$ independent of $\gamma$ such that

$$
\|u\|_{W^{2, \gamma}\left(B_{1 / 2}\right)} \leq \frac{C}{\gamma-1}\left(\|\Delta u\|_{L^{\gamma}\left(B_{2 / 3}\right)}+\|u\|_{L^{\gamma}\left(B_{2 / 3}\right)}\right) .
$$

Of course, even in the $L^{1}$ case, we have the following suboptimal estimate, which one can prove using standard estimates for harmonic functions and because the operator $\nabla N: L^{1}\left(B_{1}\right) \rightarrow L^{2, \infty}\left(B_{1}\right) \hookrightarrow L^{1}\left(B_{1}\right)$ is bounded (see Appendix A.4).

Lemma A.2. If $u \in L^{1}\left(B_{1}\right)$ is a weak solution to

$$
-\Delta u=f \in L^{1}\left(B_{1}\right),
$$

then $u \in W^{1,1}\left(B_{2 / 3}\right)$ and there exists $C<\infty$ such that

$$
\|\nabla u\|_{L^{1}\left(B_{2 / 3}\right)} \leq C\left(\|f\|_{L^{1}\left(B_{1}\right)}+\|u\|_{L^{1}\left(B_{1}\right)}\right) .
$$

We now present a theorem of Adams giving improved estimates on the Riesz potential $I_{1}$ if, in addition, we have a decay estimate on our function. Given $h \in L^{1}\left(B_{1}, \mathbb{R}^{m}\right)$ define a new function $I_{1} h$ to be the convolution of $|h|$, extended to be zero on $\mathbb{R}^{2} \backslash B_{1}$, with $|x|^{-1}$, i.e.

$$
I_{1} h(x)=\int_{B_{1}} \frac{|h(y)|}{|x-y|} d y .
$$

Lemma A.3 (Adams [1, Propositions 3.1 and 3.2]). Suppose $h \in L^{1}\left(B_{1}\right)$ and

$$
\sup _{x \in B_{1 / 2}, r \in(0,1 / 2)} r^{-\alpha} \int_{B_{r}(x)}|h|<\infty,
$$


for some $\alpha \in(0,1)$. Then

(1) $I_{1} h \in L^{q}\left(B_{1 / 4}\right)$ for any $q \in\left[1, \frac{2-\alpha}{1-\alpha}\right)$;

(2) if in addition $h \in L_{l o c}^{s}\left(B_{1}\right)$ for $s \in\left(1, \frac{2}{2-\alpha}\right)$, then $I_{1} h \in L^{s\left(\frac{2-\alpha}{1-\alpha}\right)}\left(B_{1 / 4}\right)$.

A.2. Hardy spaces. Pick $\phi \in C_{c}^{\infty}\left(B_{1}\right)$ such that $\int \phi=1$ and let $\phi_{t}(x)=t^{-2} \phi\left(\frac{x}{t}\right)$. For a distribution $f$, we say that $f$ lies in the Hardy space $\mathcal{H}^{1}\left(\mathbb{R}^{2}\right)$ if $f_{*} \in L^{1}\left(\mathbb{R}^{2}\right)$, where

$$
f_{*}(x)=\sup _{t>0}\left|\left(\phi_{t} * f\right)(x)\right|
$$

with norm $\|f\|_{\mathcal{H}^{1}\left(\mathbb{R}^{2}\right)}=\left\|f_{*}\right\|_{L^{1}\left(\mathbb{R}^{2}\right)}$. Clearly we have the continuous embedding $\mathcal{H}^{1}\left(\mathbb{R}^{2}\right) \hookrightarrow L^{1}\left(\mathbb{R}^{2}\right)$. The dual space of $\mathcal{H}^{1}\left(\mathbb{R}^{2}\right)$ is $B M O\left(\mathbb{R}^{2}\right)$ where $B M O:=\{g \in$ $\left.L_{l o c}^{1}\left(\mathbb{R}^{2}\right): \sup _{B \subset \mathbb{R}^{2}} \frac{1}{|B|} \int_{B}|g-\bar{g}|<\infty\right\}$ (see [5]).

Related to $\mathcal{H}^{1}$ is the so-called local Hardy space $h^{1}$ defined to be those functions for which

$$
f_{\tilde{*}}(x)=\sup _{0<t<1}\left|\left(\phi_{t} * f\right)(x)\right| \in L^{1}\left(\mathbb{R}^{2}\right)
$$

with corresponding norm. Again we clearly have the continuous embedding $h^{1}\left(\mathbb{R}^{2}\right)$ $\hookrightarrow L^{1}\left(\mathbb{R}^{2}\right)$. For a function $f$ defined in $B_{1}$ we say that $f \in h^{1}\left(B_{1}\right)$ if

$$
f_{\widehat{*}}(x)=\sup _{0<t<1-|x|}\left|\left(\phi_{t} * f\right)(x)\right| \in L^{1}\left(B_{1}\right) .
$$

By [15. Theorem 1.92] we know that $f \in h^{1}\left(B_{1}\right)$ if and only if for any $\varphi \in C_{c}^{\infty}\left(B_{1}\right)$ with $\int \varphi \neq 0$ there is a constant $\lambda$ such that $\varphi(f-\lambda) \in \mathcal{H}^{1}\left(\mathbb{R}^{2}\right)$, with

$$
\|\varphi(f-\lambda)\|_{\mathcal{H}^{1}\left(\mathbb{R}^{2}\right)} \leq C\|f\|_{h^{1}\left(B_{1}\right)},
$$

where $C=C(\varphi)$ and $\lambda$ is chosen such that $\int \varphi(f-\lambda)=0$.

The Hardy spaces act as replacements to $L^{1}$ in Calderón-Zygmund estimates. In particular for $f \in \mathcal{H}^{1}\left(\mathbb{R}^{2}\right)$, writing $w=N[f]$, we have the estimate (see [9])

$$
\left\|\nabla^{2} w\right\|_{L^{1}\left(\mathbb{R}^{2}\right)} \leq C\|f\|_{\mathcal{H}^{1}\left(\mathbb{R}^{2}\right)},
$$

and if $f \in h^{1}\left(\mathbb{R}^{2}\right)$, then $\nabla^{2} w \in L_{l o c}^{1}\left(\mathbb{R}^{2}\right)$ with

$$
\left\|\nabla^{2} w\right\|_{L^{1}\left(B_{1}\right)} \leq C\|f\|_{h^{1}\left(\mathbb{R}^{2}\right)} .
$$

Moreover $f \in h^{1}\left(B_{1}\right)$ implies $\nabla^{2} w \in L_{\text {loc }}^{1}\left(B_{1}\right)$. The final two assertions follow from (33), [15, Theorem 1.92] and standard elliptic estimates.

A.3. Lorentz spaces and $L \ln L$. For measurable $f$ define, for $s \geq 0$, the distribution function $\lambda(s)=|\{x:|f|(x)>s\}|$. Assuming $\lim _{s \rightarrow \infty} \lambda(s)=0$, define the nonincreasing rearrangement $f^{*}:(0, \infty) \rightarrow[0, \infty)$ by

$$
f^{*}(t):=\inf \{s \geq 0: \lambda(s) \leq t\} .
$$

Here we consider the spaces defined by:

(1) $L^{2,1}:=\left\{f: \int t^{-1 / 2} f^{*}(t) d t<\infty\right\}$,

(2) $L^{2, \infty}:=\left\{f: \sup _{t>0} t^{1 / 2} f^{*}(t)<\infty\right\}$,

(3) $L \ln L:=\left\{f: \int f^{*}(t) \ln \left(2+\frac{1}{t}\right) d t<\infty\right\}$.

The quantities above are not norms, but the spaces are all Banach spaces whose norms are equivalent to these quantities respectively. The spaces $L^{2,1}$ and $L^{2, \infty}$ are two examples of Lorentz spaces, which can be thought of as perturbations of 
the usual $L^{p}$ spaces. For example the following are all continuous embeddings (see [18]):

$$
L^{p}\left(B_{1}\right) \hookrightarrow L^{2,1}\left(B_{1}\right) \hookrightarrow L^{2}\left(B_{1}\right)=L^{2,2}\left(B_{1}\right) \hookrightarrow L^{2, \infty}\left(B_{1}\right) \hookrightarrow L^{q}\left(B_{1}\right)
$$

for all $q<2<p$. The dual space of $L^{2,1}$ is $L^{2, \infty}[10$.

For the space $L \ln L$ we have the continuous embeddings

$$
L^{p}\left(B_{1}\right) \hookrightarrow L \ln L\left(B_{1}\right) \hookrightarrow L^{1}\left(B_{1}\right)
$$

for all $p>1$. It is well known [17] that $f \in L \ln L$ if and only if its corresponding maximal function is locally integrable, where the maximal function $M_{0}(f)(x)=$ $\sup _{t>0} \frac{1}{\left|B_{t}(x)\right|} \int_{B_{t}(x)}|f|$. By extension of $f$ by zero and comparison of the functions $M_{0}(f)$ and $f_{\tilde{*}}$ we see that the following embedding is continuous:

$$
L \ln L\left(B_{1}\right) \hookrightarrow h^{1}\left(\mathbb{R}^{2}\right) .
$$

In fact, if $f \in L \ln L\left(B_{1}\right)$, then $f-\bar{f} \in \mathcal{H}^{1}\left(\mathbb{R}^{2}\right)$ with $\|f-\bar{f}\|_{\mathcal{H}^{1}\left(\mathbb{R}^{2}\right)} \leq C\|f\|_{h^{1}\left(\mathbb{R}^{2}\right)} \leq$ $C\|f\|_{L \ln L\left(B_{1}\right)}$, where

$$
f-\bar{f}:=\left\{\begin{array}{cl}
f-\frac{1}{\left|B_{1}\right|} \int_{B_{1}} f & \text { in } B_{1} \\
0 & \text { in } \mathbb{R}^{2} \backslash B_{1} .
\end{array}\right.
$$

A.4. Embeddings and estimates. Listed below are some important miscellaneous results involving Sobolev spaces and the spaces mentioned above.

(1) The embedding $W^{1,1}\left(\mathbb{R}^{2}\right) \hookrightarrow L^{2,1}\left(\mathbb{R}^{2}\right)$ is continuous 9 .

(2) Given $u, v \in W^{1,2}\left(\mathbb{R}^{2}\right)$, then $\nabla u . \nabla^{\perp} v \in \mathcal{H}^{1}\left(\mathbb{R}^{2}\right)$ with $\left\|\nabla u . \nabla^{\perp} v\right\|_{\mathcal{H}^{1}\left(\mathbb{R}^{2}\right)} \leq$ $C\|\nabla u\|_{L^{2}\left(\mathbb{R}^{2}\right)}\|\nabla v\|_{L^{2}\left(\mathbb{R}^{2}\right)}$. (See [2].)

(3) By (1) and the estimates from Appendices A.1 and A.2 on the Newtonian potential we have that the operators $\nabla N: h^{1}\left(\mathbb{R}^{2}\right) \rightarrow L_{l o c}^{2,1}\left(\mathbb{R}^{2}\right)$ and $\nabla N:$ $\mathcal{H}^{1}\left(\mathbb{R}^{2}\right) \rightarrow L_{\text {loc }}^{2,1}\left(\mathbb{R}^{2}\right)$ are bounded.

(4) $\nabla N: L^{1}\left(B_{1}\right) \rightarrow L^{2, \infty}\left(B_{1}\right)$ is a bounded operator; this follows by standard estimates on convolutions and the fact that $\nabla \Gamma \in L^{2, \infty}$.

(5) The embedding $W^{1,2}\left(\mathbb{R}^{2}\right) \hookrightarrow B M O\left(\mathbb{R}^{2}\right)$ is continuous by Poincaré's inequality.

A.5. Rivière's gauge. The key result from Rivière's work that we will need is the existence of the following perturbation of Coulomb's gauge.

Lemma A.4 (Rivière). Suppose $\Omega \in L^{2}\left(B_{1}, s o(m) \otimes \mathbb{R}^{2}\right)$. Then there exists $\epsilon=\epsilon(m)>0$ such that if $\|\Omega\|_{L^{2}\left(B_{1}\right)} \leq \epsilon$ we can find $A \in W^{1,2}\left(B_{1}, G L_{m}(\mathbb{R})\right) \cap$ $L^{\infty}\left(B_{1}, G L_{m}(\mathbb{R})\right), B \in W^{1,2}\left(B_{1}, g l_{m}(\mathbb{R})\right)$ and $C=C(m)<\infty$, where

$$
\nabla A-A \Omega=\nabla^{\perp} B
$$

and

$$
\|\nabla A\|_{L^{2}\left(B_{1}\right)}+\|\nabla B\|_{L^{2}\left(B_{1}\right)}+\| \operatorname{dist}\left(A, S O(m)\left\|_{L^{\infty}\left(B_{1}\right)} \leq C\right\| \Omega \|_{L^{2}\left(B_{1}\right)} .\right.
$$

A.6. Weak convergence of measures and functions of bounded variation. We consider the space of functions of bounded variation $B V(B)$ for any ball $B \subset \mathbb{R}^{2}$. $B V$ is defined by

$$
B V(B)=\left\{V \in L^{1}(B): \int_{B}|\nabla V|:=\sup _{\phi \in C_{0}^{1}\left(B, \mathbb{R}^{2}\right)\|\phi\|_{L^{\infty} \leq 1}} \int_{B} V \operatorname{div} \phi<\infty\right\} .
$$


In other words it is the space of functions whose distributional derivatives are signed Radon measures with finite total mass. This is a Banach space with norm $\|V\|_{B V(B)}=\|V\|_{L^{1}(B)}+\int_{B}|\nabla V|$. It is easy to see that we have the continuous embedding $W^{1,1} \hookrightarrow B V$; moreover in two dimensions we have the continuous embedding $B V(B) \hookrightarrow L^{2}(B)$ and the compact embeddings $B V(B) \hookrightarrow L^{p}(B)$ for any $p<2$ (see for instance [18]).

We also use the standard weak-* compactness available in the space of signed Radon measures with finite total mass, denoted $M$.

The proof of the next lemma is essentially taken from [3, Theorem 9] and is similar to that stated in [11. For an integrable function $k$ we implicitly view it as both a function and a measure, i.e. $k=k d x$.

Lemma A.5. Suppose $\left\{V_{n}\right\} \subset B V(B)$ is a bounded sequence and $B \subset \mathbb{R}^{2}$ is an open ball. Then there exist at most countable $\left\{x_{j}\right\} \subset B$ and $\left\{a_{j}>0\right\}$ (where $\sum_{j} a_{j}<\infty$ ) and $V \in B V(B)$ such that (up to a subsequence)

$$
V_{n}^{2} \rightarrow V^{2}+\sum_{j} a_{j} \delta_{x_{j}}
$$

weakly in $M(B)$.

Proof. Since $\left\{V_{n}\right\} \subset B V(B)$ is a bounded sequence, there exists $V \in L^{2}$ such that (up to a subsequence) $V_{n} \rightarrow V$ strongly in $L^{p}$ for all $p<2$ and $V_{n} \rightarrow V$ weakly in $L^{2}$. Also $\left\{\nabla V_{n}\right\} \subset M(B)$ is bounded so (again up to a subsequence) $\nabla V_{n} \rightarrow \lambda$ (a vector-valued measure $) \in M(B)$. In particular, for all $\phi \in C_{c}^{1}\left(B, \mathbb{R}^{2}\right)$,

$$
\begin{aligned}
\int \phi \cdot d \lambda & =\lim _{n \rightarrow \infty} \int \phi \cdot \nabla V_{n} d x \\
& =-\lim _{n \rightarrow \infty} \int \operatorname{div}(\phi) V_{n} d x \\
& =-\int \operatorname{div}(\phi) V d x .
\end{aligned}
$$

In other words $V \in B V(B)$ and $\nabla V=\lambda$.

Now set $g_{n}:=V_{n}-V$. Note that $\left|\nabla g_{n}\right| \in M(B)$ is bounded, so for a subsequence $\left|\nabla g_{n}\right| \rightarrow \mu \in M(B)$, where $\mu$ is nonnegative. Similarly (up to a subsequence) $g_{n}^{2} \rightarrow \nu \in M(B)$, where $\nu$ is also nonnegative. We have that for all $\phi \in C_{c}^{1}(B)$, $\phi g_{n} \in B V(B)$ and by the continuous embedding $B V(B) \hookrightarrow L^{2}(B)$ we have

$$
\left(\int\left(\phi g_{n}\right)^{2} d x\right)^{1 / 2} \leq C \int\left|\nabla\left(\phi g_{n}\right)\right| d x
$$

and since $g_{n} \rightarrow 0$ in $L^{1}$, taking limits gives

$$
\left(\int \phi^{2} d \nu\right)^{1 / 2} \leq C \int|\phi| d \mu .
$$

Taking $\phi$ to be an approximation to the characteristic function on $B_{r}(x) \subset B$ we get

$$
\nu\left(B_{r}(x)\right) \leq C\left(\mu\left(B_{r}(x)\right)\right)^{2}
$$

for all $B_{r}(x) \subset B$, and in particular $\nu \ll \mu$. 
By standard results for differentiation of measures (see e.g. [4, $\S 1.6$ Theorem 2]), for any Borel set $E \subset B$,

$$
\nu(E)=\int_{E} D_{\mu} \nu d \mu
$$

where $D_{\mu} \nu=\lim _{r \downarrow 0} \frac{\nu\left(B_{r}(x)\right)}{\mu\left(B_{r}(x)\right)}$ is a $\mu$-integrable function (this limit exists $\mu$-almost everywhere).

Since $\mu$ is a finite, positive Radon measure, there are at most countable points $\left\{x_{j}\right\}$ such that $\mu\left(\left\{x_{j}\right\}\right)>0$, and if $\mu(\{x\})=0$, then

$$
D_{\mu} \nu(x)=\lim _{r \downarrow 0} \frac{\nu\left(B_{r}(x)\right)}{\mu\left(B_{r}(x)\right)} \leq C \lim _{r \downarrow 0} \mu\left(B_{r}(x)\right)=0 .
$$

Letting $X:=\bigcup_{j}\left\{x_{j}\right\}$ we have $D_{\mu} \nu=0 \mu$-almost everywhere on $B \backslash X$. Hence $D_{\mu} \nu$ is a simple function; therefore for Borel $E \subset B$,

$$
\nu(E)=\int_{E} D_{\mu} \nu d \mu=\sum_{\left\{j: x_{j} \in E\right\}} D_{\mu} \nu\left(x_{j}\right) \mu\left(\left\{x_{j}\right\}\right) .
$$

Setting $a_{j}:=D_{\mu} \nu\left(x_{j}\right) \mu\left(\left\{x_{j}\right\}\right)$ we have $\nu=\sum_{j} a_{j} \delta_{x_{j}}$. Now, for $\phi \in C_{c}^{0}(B)$,

$$
\begin{aligned}
\sum_{j} a_{j} \phi\left(x_{j}\right) & =\lim _{n \rightarrow \infty} \int g_{n}^{2} \phi d x \\
& =\lim _{n \rightarrow \infty} \int\left(V_{n}-V\right)^{2} \phi d x \\
& =\lim _{n \rightarrow \infty}\left(\int\left(V_{n}^{2}-V^{2}\right) \phi d x+2 \int V\left(V-V_{n}\right) \phi d x\right)
\end{aligned}
$$

where the last term vanishes in the limit since $V_{n} \rightarrow V$ weakly in $L^{2}$.

Lemma A.6 (Corollary of Lemma A.5). Suppose $\left\{V_{n}\right\}$ is as in Lemma A.5. If

$$
\lim _{r \downarrow 0} \limsup _{n \rightarrow \infty}\left\|V_{n}\right\|_{L^{2}\left(B_{r}(x)\right)}=0
$$

for all $x \in B$, then

$$
V_{n} \rightarrow V
$$

strongly in $L_{l o c}^{2}(B)$ (same $V$ as in Lemma A.5).

Proof. First we apply Lemma A.5 and viewing $\left|V_{n}\right|^{2} d x$ as a sequence in $M(B)$ we notice that the condition $\lim _{r \downarrow 0} \lim \sup _{n \rightarrow \infty}\left\|V_{n}\right\|_{L^{2}\left(B_{r}(x)\right)}=0$ simply says that $V_{n}^{2} \rightarrow V^{2}$ weakly in $M(B)$. Therefore, given any open ball $B_{r}(x) \Subset B$ we can apply standard results for Radon measures $([4, \S 1.9$, Theorem 1]) to conclude that (since $\left.\int_{\partial B_{r}(x)}|V|^{2} d x=0\right)\left\|V_{n}\right\|_{L^{2}\left(B_{r}(x)\right)} \rightarrow\|V\|_{L^{2}\left(B_{r}(x)\right)}$ for all $B_{r}(x) \Subset B$. Hence

$$
\begin{aligned}
\int_{B_{r}(x)}\left(V-V_{n}\right)^{2} d x & =\int_{B_{r}(x)}\left(V_{n}^{2}-V^{2}\right) d x+2 \int_{B_{r}(x)} V\left(V-V_{n}\right) d x \\
& \rightarrow 0 \quad \text { as } n \rightarrow \infty
\end{aligned}
$$

since $V_{n} \rightarrow V$ weakly in $L^{2}$. Therefore $V_{n} \rightarrow V$ strongly in $L_{l o c}^{2}(B)$. 
A.7. Absorption lemma. Special cases of the following lemma are widely used in regularity theory.

Lemma A.7 (Leon Simon [16, $\S 2.8$, Lemma 2]). Let $B_{\rho}(y) \subset \mathbb{R}^{2}$ be any ball, $k \in \mathbb{R}$, $\Gamma>0$, and let $\varphi$ be any $[0, \infty)$-valued convex subadditive function on the collection of convex subsets of $B_{\rho}(y)$; thus $\varphi(A) \leq \sum_{j=1}^{N} \varphi\left(A_{j}\right)$ whenever $A, A_{1}, A_{2}, \ldots, A_{N}$ are convex subsets of $B_{\rho}(y)$ with $A \subset \bigcup_{j=1}^{N} A_{j}$. There is $\epsilon_{0}=\epsilon_{0}(k)$ such that if

$$
\sigma^{k} \varphi\left(B_{\sigma / 2}(z)\right) \leq \epsilon_{0} \sigma^{k} \varphi\left(B_{\sigma}(z)\right)+\Gamma
$$

whenever $B_{2 \sigma}(z) \subset B_{\rho}(y)$, then there exists some $C=C(k)<\infty$ such that

$$
\rho^{k} \varphi\left(B_{\rho / 2}(y)\right) \leq C \Gamma .
$$

\section{ACKNOWLEDGMENTs}

Both authors were supported by The Leverhulme Trust. The second author would like to thank Paweł Strzelecki for useful discussions. Thanks also to the referee for useful comments.

\section{REFERENCES}

[1] David R. Adams. A note on Riesz potentials. Duke Math. J., 42(4):765-778, 1975. MR.0458158(56:16361)

[2] R. Coifman, P.-L. Lions, Y. Meyer, and S. Semmes. Compensated compactness and Hardy spaces. J. Math. Pures Appl. (9), 72(3):247-286, 1993. MR1225511 (95d:46033)

[3] Lawrence C. Evans. Weak convergence methods for nonlinear partial differential equations, CBMS Regional Conference Series in Mathematics 74, 1990. MR1034481 (91a:35009)

[4] Lawrence C. Evans and Ronald F. Gariepy. Measure theory and fine properties of functions. Studies in Advanced Mathematics. CRC Press, Boca Raton, FL, 1992. MR.1158660 (93f:28001)

[5] C. Fefferman and E. M. Stein. $H^{p}$ spaces of several variables. Acta Math., 129:137-193, 1972. MR0447953(56:6263)

[6] Yuxin Ge. Estimations of the best constant involving the $L^{2}$ norm in Wente's inequality and compact $H$-surfaces in Euclidean space. ESAIM Control Optim. Calc. Var., 3:263-300, 1998. MR.1634837 (2000a:58038)

[7] David Gilbarg and Neil S. Trudinger. Elliptic partial differential equations of second order. Classics in Mathematics. Springer-Verlag, Berlin, 2001. MR 1814364 (2001k:35004)

[8] F. Hélein. Régularité des applications faiblement harmoniques entre une surface et une variété riemannienne. C. R. Acad. Sci. Paris Sér. I Math., 312:591-596, 1991. MR1101039 (92e:58055)

[9] Frédéric Hélein. Harmonic maps, conservation laws and moving frames, Cambridge Tracts in Mathematics 150. Cambridge University Press, second edition, 2002. MR1913803 (2003g:58024)

[10] Richard A. Hunt. On $L(p, q)$ spaces. Enseignement Math. (2), 12:249-276, 1966. MR.0223874 $(36: 6921)$

[11] J. Li and X. Zhu. Small energy compactness for approximate harmonic mappings. Preprint, 2009.

[12] Tristan Rivière. Conservation laws for conformally invariant variational problems. Invent. Math., 168(1):1-22, 2007. MR2285745 (2008d:58010)

[13] Tristan Rivière. Integrability by compensation in the analysis of conformally invariant problems. Minicourse Notes, 2009.

[14] M. Rupflin, An improved uniqueness result for the harmonic map flow in two dimensions. Calc. Var. 33:329-341, 2008. MR2429534(2009g:53106)

[15] Stephen Semmes. A primer on Hardy spaces, and some remarks on a theorem of Evans and Müller. Comm. Partial Differential Equations, 19(1-2):277-319, 1994. MR1257006 (94j:46038) 
[16] Leon Simon. Theorems on regularity and singularity of energy minimizing maps. Lectures in Mathematics ETH Zürich. Birkhäuser Verlag, Basel, 1996. MR1399562 (98c:58042)

[17] E. M. Stein. Note on the class L log L. Studia Math., 32:305-310, 1969. MR0247534 (40:799)

[18] William P. Ziemer. Weakly differentiable functions, volume 120 of Graduate Texts in Mathematics. Springer-Verlag, New York, 1989. MR 1014685 (91e:46046)

Mathematics Institute, University of Warwick, Coventry, CV4 7AL, United Kingdom Current address: Department of Mathematics, Imperial College London, South Kensington Campus, London, SW7 2AZ, United Kingdom

Mathematics Institute, University of Warwick, Coventry, CV4 7AL, United Kingdom 